\title{
Phase diagrams for sonoluminescing bubbles
}

\author{
Sascha Hilgenfeldt and Detlef Lohse \\ Fachbereich Physik der Universität Marburg, Renthof 6, 35032 Marburg, Germany \\ Michael P. Brenner \\ Department of Mathematics, Massachusetts Institute of Technology, Cambridge, Massachusetts 02139
}

(Received 29 May 1996; accepted 23 July 1996)

\begin{abstract}
Sound driven gas bubbles in water can emit light pulses. This phenomenon is called sonoluminescence (SL). Two different phases of single bubble SL have been proposed: diffusively stable and diffusively unstable SL. We present phase diagrams in the gas concentration versus forcing pressure state space and also in the ambient radius versus gas concentration and versus forcing pressure state spaces. These phase diagrams are based on the thresholds for energy focusing in the bubble and two kinds of instabilities, namely (i) shape instabilities and (ii) diffusive instabilities. Stable SL only occurs in a tiny parameter window of large forcing pressure amplitude $P_{a} \sim 1.2-1.5 \mathrm{~atm}$ and low gas concentration of less than $0.4 \%$ of the saturation. The upper concentration threshold becomes smaller with increased forcing. Our results quantitatively agree with experimental results of Putterman's UCLA group on argon, but not on air. However, air bubbles and other gas mixtures can also successfully be treated in this approach if in addition (iii) chemical instabilities are considered. All statements are based on the Rayleigh-Plesset ODE approximation of the bubble dynamics, extended in an adiabatic approximation to include mass diffusion effects. This approximation is the only way to explore considerable portions of parameter space, as solving the full PDEs is numerically too expensive. Therefore, we checked the adiabatic approximation by comparison with the full numerical solution of the advection diffusion PDE and find good agreement. (C) 1996 American Institute of Physics. [S1070-6631(96)02011-9]
\end{abstract}

\section{INTRODUCTION}

\section{A. The phenomenon}

A gas bubble levitated in a strong acoustic field

$P(t)=P_{a} \cos \omega t$

can emit bursts of light so intense as to be observable by the naked eye. ${ }^{1-8}$ Here, $P_{a}$ is the forcing pressure amplitude and $\omega / 2 \pi$ the frequency of the forcing field. This phenomenon is called single bubble sonoluminescence (SL). The light pulse is shorter than 50 ps. $^{2,9}$ Precise experiments by Putterman's group at $\mathrm{UCLA}^{2-7}$ have revealed many surprising and intriguing properties of sonoluminescing bubbles. SL only occurs in a narrow parameter range. The adjustable experimental parameters we focus on here are the forcing pressure amplitude $P_{a}$ and the gas concentration $c_{\infty}$ far from the bubble. Single bubble SL is found only for large (compared to the ambient pressure $P_{0}$ ) forcing pressure $P_{a} \sim 1.2-1.5$ atm and small (compared to the saturation $c_{0}$ ) gas concentration $c_{\infty}$.

We report the results on argon bubbles first. Two distinct phases of single bubble SL have been proposed on account of experimental observations: ${ }^{5}$ (i) Unstable $S L$ occurs in the concentration range $c_{\infty} / c_{0} \approx 6 \%-26 \%$. This phase is characterized by an increase of the relative phase of light emission with respect to the driving pressure on the slow diffusive time scale $\sim 1 \mathrm{~s}$, followed by a rapid breakdown and another subsequent increase. The light intensity itself behaves in the same way and the bubble is reported to be dancing or jiggling. ${ }^{1,5}$ This state of SL is also unstable in the sense that often all of a sudden the bubble dies. (ii) Stable $S L$ occurs in argon bubbles at very low gas concentrations $c_{\infty} / c_{0} \sim 0.4 \%$. The diffusively stable state is characterized by the constancy of the relative phase of light emission over billions of cycles. The same is true for the light intensity.

For air bubbles the same two phases exist, however, for gas concentrations about two orders of magnitude larger than for argon bubbles: Stable SL is observed for $c_{\infty}^{\text {air }} / c_{0} \sim 10 \%-20 \%,{ }^{5}$ unstable SL for even higher concentrations.

Another controllable parameter is the temperature of the liquid. Upon decreasing the temperature of the ambient water from room temperature to slightly above freezing, the light intensity may increase by two orders of magnitude. ${ }^{3}$ Abrupt transitions in the light intensity with the liquid temperature are found for SL in nonaqueous fluids. ${ }^{10}$ Using different fluids (but the same gas species) also results in great differences in SL intensity. ${ }^{10,11}$

\section{B. A hydrodynamic approach}

The goal of this paper is to figure out which features of SL can be accounted for in a purely hydrodynamic approach ${ }^{6}$ and to which extent they may reflect other, nonhydrodynamic effects, e.g., chemistry.

Our main result is the phase diagram Fig. 1 in the $P_{a}-c_{\infty}$ phase space. It is obtained from hydrodynamic calculations of the bubble dynamics and the fluid dynamical and diffusive processes outside the bubble. For given forcing pressure amplitude $P_{a}$ and gas concentration $c_{\infty}$ we predict with this diagram whether the bubble is in the diffusively unstable SL state, the diffusively stable SL state, or in no SL state at all.

Besides the forcing pressure amplitude $P_{a}$ and the gas 


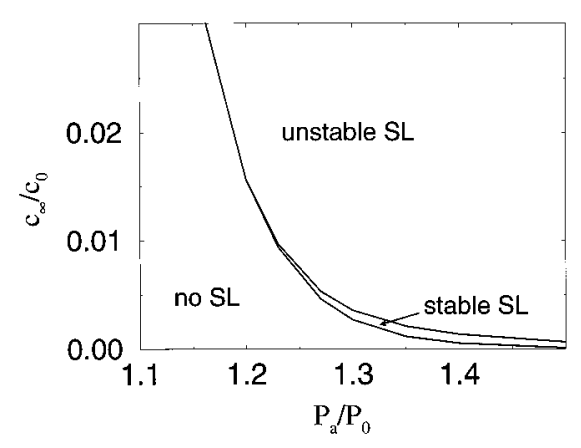

FIG. 1. Phase diagram in the $c_{\infty} / c_{0}$ vs $P_{a} / P_{0}$ parameter space. The three phases represent stable SL, unstable SL, and no SL. For lucidity we do not draw the upper and the right borderline of the unstable SL regime toward a no SL regime as they are less precisely defined.

concentration $c_{\infty}$, two further parameters have to be addressed here: The frequency $\omega / 2 \pi$ of the forcing field and the ambient radius $R_{0}$ of the bubble, i.e., the bubble radius at ambient normal conditions of $P_{0}=1 \mathrm{~atm}$ and $293 \mathrm{~K}$. The frequency is set to a fixed value so that the forcing acoustic field (1) corresponds to a resonance of the container, in order that the bubble remains trapped in a pressure antinode. All of the analysis corresponds to $\omega=2 \pi 26.5 \mathrm{kHz}$ as applied in Barber et al.'s experiment; ${ }^{5}$ the period of the forcing field is thus $T=2 \pi / \omega=38 \mu \mathrm{s}$.

The ambient radius $R_{0}$ of the bubble is not an adjustable parameter but the system chooses $R_{0}$ dynamically. The approach followed here includes this (diffusive) dynamics, so that we can predict the ultimate ambient radius. Our results are consistent with Mie scattering radius measurements. ${ }^{5}$

What are the necessary requirements for SL to occur? First, the bubble has to be stable toward shape oscillations. ${ }^{12-17}$ We have identified three types of shape instability: Two instabilities of a parametric type acting on relatively slow time scales of $\sim 1-100 \mu \mathrm{s}$. As discussed below, these instabilities are quite gentle and the bubble can survive them by pinching off microbubbles. This pinch off causes the aforementioned break in the relative phase of light emission in the unstable SL state $^{5}$ and the recoil of the remaining bubble is the origin of the observed "jiggling" or "dancing" of the bubble. The third type of shape instability, however, acts on a very short time scale $\lesssim 10^{-9}$ s. It is so violent that it ejects the bubble from the trapping sound field. We call it the Rayleigh-Taylor instability as it occurs when gas from inside the bubble is accelerated towards the fluid.

What is the energy focusing process in the bubble? Many speculations abound in literature. ${ }^{18,19} \operatorname{Jarman}^{20}$ (for multi bubble SL) and later Greenspan and $\mathrm{Nadim}^{21}$ and $\mathrm{Wu}$ and Roberts ${ }^{22}$ suggest that shocks detach during the compression of the bubble and focus to the center of the bubble, thereby compressing the gas so strongly that light can be emitted, either by ionization and subsequent bremsstrahlung ${ }^{22,6}$ or by blackbody radiation. ${ }^{11}$ In Ref. 23 we suggest an alternate energy focusing mechanism. The idea is that the bubble acts as a driven acoustic resonator which switches on when the damping losses through viscosity and acoustic radiation are smaller than the energy input during the collapse. The acoustic energy accumulates and finally results in light pulses. This theory offers an explanation for the possibility of the above mentioned abrupt transition in the SL intensity with increasing temperature, ${ }^{10}$ as well as for the dependence of the light intensity on different liquids and gases. We refer to Ref. 23 for a detailed discussion.

In this paper we take as criterion for energy focusing and the resulting light production that the Mach number of the bubble wall (with respect to the speed of sound in the gas) has to be larger than one. ${ }^{24}$ This criterion corresponds to the onset of SL within conventional shock theories ${ }^{20-22}$ and within our alternate energy focusing mechanism. ${ }^{23}$ Together with the requirement of bubble stability, it gives the boundary of the stable SL regime of Fig. 1. For argon bubbles the narrow parameter range where stable SL exists is in agreement with experiments of Barber et al..$^{5}$ A qualitative argument for this agreement was previously given by Löfstedt et al. ${ }^{7}$

\section{Chemical instabilities}

For air bubbles there are severe deviations between the hydrodynamically calculated phase space diagram and the experimental measurements. ${ }^{5}$ The parameter regime where stable air bubbles should exist is close to that of argon; however, experimentally stable SL is found for gas concentrations as large as $c_{\infty}^{\text {air }} / c_{0} \sim 20 \% .^{5}$ Because of this discrepancy, Löfstedt et al. hypothesize ${ }^{7}$ a " "yet unidentified mass ejection mechanism.' In Ref. 25 we have suggested that this mechanism is chemical. Indeed, when considering besides (i) shape instabilities and (ii) diffusive instabilities also (iii) chemical instabilities, our results can be extended to gas mixtures and are then in quantitative agreement with the UCLA experiments, as shown in detail in Ref. 26. The idea is that because of the high temperatures achieved in the bubble nitrogen and/or oxygen is destroyed and reacts to $\mathrm{NO}_{3}^{-}, \mathrm{NO}_{2}^{-}$, and/or $\mathrm{NH}_{4}^{+}$and only pure argon remains in the bubble. Thus for air which contains about $1 \%$ argon the gas concentrations $c_{\infty}^{\text {air }}$ in water have to be about two orders of magnitude higher than for pure argon. The central parameter is thus the argon (or inert gas) concentration $c_{\infty}^{\mathrm{Ar}}=q c_{\infty}^{\text {mixture }}$ in the dissolved gas. Here, $q$ is the percentage of argon in the mixture; for air $q=0.01=1 \%$. The nitrogen dissociation theory suggests that when adjusting $q$ properly, no degasing is necessary any more. $^{25,26}$

In this paper we work out the basics of our hydrodynamic approach and restrict ourselves to pure argon bubbles for which no chemical instabilities (i.e., reactions) can occur. However, by considering the chemical instabilities properly, ${ }^{25,26}$ our results here can directly be extended to any gas mixture and are found to agree with the UCLA experiments.

\section{Necessary approximations}

How can we examine the huge multi-dimensional parameter space $\left(P_{a}, c_{\infty}\right.$, and $\left.R_{0}\right)$ ? Given that the dynamics involve time scales spanning eleven orders of magnitude (from the time scale of the light flash $(<50 \mathrm{ps})$ to the diffusive time scale $(\sim 1 \mathrm{~s})$ ), it is necessary to make approxima- 
tions in modeling the hydrodynamics of sonoluminescence. The full hydrodynamic problem involves solving the threedimensional (3-D) Navier-Stokes equations both inside and outside the bubble, coupled with equations of heat transfer and gas transfer, accompanied by the correct boundary conditions at the interface and at infinity and the equations of state. Moreover, at least in principle the radiation fields need to be coupled to the fluid. This set of equations must be studied not only as a function of parameters but also over millions of oscillation periods of the bubble; it should be emphasized that the relevant question for sonoluminescence experiments is not the transient that occurs for the first few cycles but rather the nature of the long time limit. This complete formulation is both computationally and theoretically intractable. In order to make progress, the problem must be simplified. To date, two different avenues have been pursued.

The first approximation was proposed long ago by Lord Rayleigh $^{27}$ and elaborated upon by Plesset, ${ }^{28}$ Taylor, ${ }^{29}$ Lauterborn, ${ }^{30}$ Prosperetti ${ }^{31,16}$ and others, ${ }^{32}$ in the context of studies of cavitation. The idea is to consider the bubble as a perfectly spherical cavity, with the pressure inside the bubble having no spatial variations. The temporal variation of the pressure follows from an equation of state. In this approach the full dynamics is reduced to the Rayleigh-Plesset (RP) ODE. ${ }^{33}$ This formulation allows very long time calculations of the bubble dynamics, but it completely ignores the dynamics inside the bubble producing the light. Later on, Plesset, ${ }^{12}$ Strube, ${ }^{15}$ and Prosperetti ${ }^{16}$ extended this type of approach to deal with shape oscillations while Epstein and Plesset, ${ }^{34}$ Eller and Crum, ${ }^{35,14}$ Crum and Cordry ${ }^{36}$ and finally Fyrillas and Szeri ${ }^{37}$ and Löfstedt et al. ${ }^{7}$ included diffusive effects. We call this approach the RP-SL-bubble approach. Clearly, shock formation ${ }^{20,21}$ or the building up of the acoustic waves $^{23}$ inside the bubble will modify the dynamics of $R(t)$, because strictly speaking, the RP equation only holds for a Mach number smaller than one. But it is our belief that the results of this paper are robust towards the resulting changes and it is only within this RP-SL bubble approach that the exploration of the full SL parameter space and the calculation of phase diagrams are currently manageable. Full numerical simulations as in Refs. 38 and 39 are by far numerically too expensive to do such an analysis.

The second type of approximation traditionally made is complementary to the first, and focuses on the interior of the bubble and above mentioned shock formation processes. ${ }^{21,22,39,40}$ The spherically symmetric gas dynamics equations are solved inside the bubble and coupled to the Rayleigh-Plesset equation. Simplifications are typically employed in modeling the gas dynamics, for example neglecting heat and viscous dissipation. These calculations can only be carried out for a few oscillation periods, and thus are not able to resolve cumulative effects building up over many oscillations.

\section{E. The Rayleigh-Plesset equation}

The Rayleigh-Plesset (RP) equation, ${ }^{6,27,30}$ on which the entire analysis of this paper is based, describes the dynamics of the bubble radius,

$$
\begin{aligned}
R \ddot{R}+\frac{3}{2} \dot{R}^{2}= & \frac{1}{\rho_{w}}\left(p(R, t)-P(t)-P_{0}\right)+\frac{R}{\rho_{w} c_{w}} \\
& \times \frac{d}{d t}(p(R, t)-P(t))-4 \nu \frac{\dot{R}}{R}-\frac{2 \sigma}{\rho_{w} R} .
\end{aligned}
$$

Typical parameters for an argon bubble in water at room temperature are the surface tension $\sigma=0.073 \mathrm{~kg} / \mathrm{s}^{2}$, the water viscosity $\nu=10^{-6} \mathrm{~m}^{2} / \mathrm{s}$, density $\rho_{w}=1000 \mathrm{~kg} / \mathrm{m}^{3}$, and speed of sound $c_{w}=1481 \mathrm{~m} / \mathrm{s}$. The driving frequency of the acoustic field is $\omega / 2 \pi=26.5 \mathrm{kHz}$ and the external pressure $P_{0}=1 \mathrm{~atm}$. These parameters show a weak dependence on the temperature of water which is assumed to be constant throughout. We assume that the pressure inside the bubble varies according to

$$
p(R(t))=P_{0}\left(\frac{R_{0}^{3}-h^{3}}{R^{3}(t)-h^{3}}\right)^{\gamma},
$$

where $h=R_{0} / 8.86$ is the hard core van der Waals radius for argon bubbles. ${ }^{6}$ The exponent $\gamma$ is the effective polytropic exponent of the gas. Plesset and Prosperetti ${ }^{31}$ calculated how it depends on the (thermal) Péclet number $\mathrm{Pe}=R_{0}^{2} \omega / \kappa$ which gives the ratio between the bubble length scale $R_{0}$ (which we take as $\approx 5 \mu \mathrm{m}$ for the estimates in this paragraph) and the thermal diffusion length $\sqrt{\kappa / \omega}$. The thermal diffusivity $\kappa$ for argon is $\kappa \approx 2 \times 10^{-5} \mathrm{~m}^{2} / \mathrm{s}$, which yields $\mathrm{Pe} \approx 0.2$ and according to Fig. 1 of Ref. 31, the effective polytropic exponent $\gamma=1$. As discussed below, the RP equation contains much smaller time scales than $\omega^{-1}$. One could therefore argue that these smaller time scales may enter into the calculation of Pe, so that the frequency $\omega$ should be replaced by $|\dot{R}| / R$. This estimate would lead to $\operatorname{Pe}(t)$ as large as $10^{4}$ at instants of rapid bubble wall movement which implies $\gamma \approx 5 / 3$ for argon. However, since $\operatorname{Pe}(t) \gg 1$ only holds in very small time intervals $\sim 1 \mathrm{~ns}$, the global dynamics are not affected by setting the effective polytropic exponent $\gamma=1$ uniformly in time. Note that with $\gamma=1$ Eq. (3) should not be thought of as an equation of state but rather as a process equation parametrizing the isothermal conditions at the bubble wall, induced by the large heat capacity of water. The choice of $\gamma=1$ is confirmed by the full numerical simulations of Vuong and $\mathrm{Szeri}^{38}$ and by the approximation of Kamath et $\mathrm{l}^{41}$ Note that, as a consequence, there are heat fluxes back and forth across the bubble wall.

Another approximation in (2) and in the dynamical equation below for the nonspherical distortions is that translational movements of the bubble are not taken into account. If present they may cause further instability, but the experiments in which the bubble is fixed in the center of the cell seem to justify that we neglect translational motion.

The radius $R(t)$ corresponding to the forcing pressure (1) with $P_{a}=1.15 \mathrm{~atm}$ is shown in Fig. 2(b). Four time scales are hidden in the (linearized) RP equation: The period $T=38 \mu \mathrm{s}$ of the external forcing $P(t)$ (Fig. 2(a)), the intrinsic frequency $\sqrt{3 \gamma P_{0} /\left(\rho_{w} R_{0}^{2}\right)} / 2 \pi \approx(1.8 \mu \mathrm{s})^{-1}$ of the oscillating bubble which is the frequency of the afterbounces, the time scale of viscous damping $R_{0}^{2} / \nu \approx 25 \mu \mathrm{s}$, and the duration $\sim 0.1-1 \mathrm{~ns}$ of the bubble collapse, estimated in Ref. 6. A fifth time scale, determined by the surface 


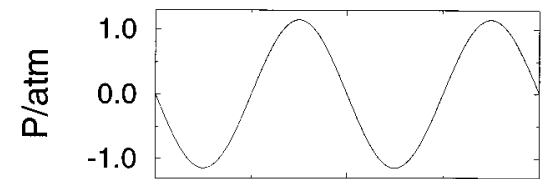

(a)

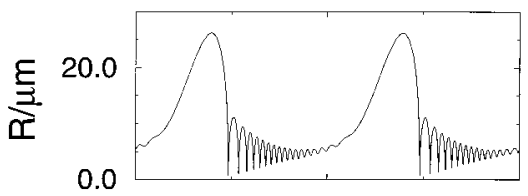

(b)

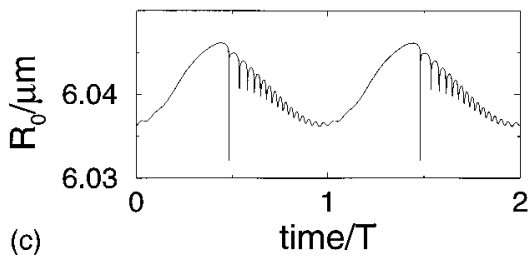

FIG. 2. (a) Forcing pressure $P(t)=P_{a} \cos \omega t, P_{a}=1.15$ atm for two cycles and the corresponding (b) $R(t)$ and (c) $R_{0}(t)$. The bubble is near an equilibrium state. The gas concentration is $c_{\infty} / c_{0}=0.035$.

tension $\sqrt{R_{0}^{3} \rho_{w} / \sigma} \approx 1.3 \mu \mathrm{s}$ is only important for large $\sigma$ or small $R$; for typical $R_{0}$, it only slightly changes the intrinsic time scale which is of the same order of magnitude.

Also diffusive processes can be understood within the

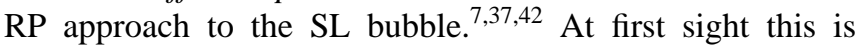
surprising because there is no diffusive time scale in the RP equation. For a qualitative understanding why this works nevertheless, look at the bubble radius $R(t)$ (Fig. 2(b)), resulting from (2). For large $R(t)$ the pressure inside the bubble will be low and gas diffuses into the bubble (rectified diffusion). For small $R(t)$, on the other hand, the bubble will shrink because of the enormous pressure inside. ${ }^{43}$ This concept was made quantitative by Fyrillas and Szeri ${ }^{37}$ and Löfstedt et al. ${ }^{7}$ The main idea is a separation of the slow diffusive time scale from all time scales in (2). We therefore call this approach the adiabatic approximation of diffusion. As we will see the balance between growth and shrinking is very delicate. In Fig. 2(c) we present the ambient radius (calculated in Section V) $R_{0}(t)$ which represents the mass $m=4 \pi R_{0}^{3} \rho_{0} / 3$ of the bubble, with the ambient density $\rho_{0} \approx 1.6 \mathrm{~kg} / \mathrm{m}^{3}$ for argon. It corresponds to the $R(t)$ curve in Fig. 2(b). The exchange processes between the bubble and the liquid can be very violent. In Fig. 2(c) the bubble's mass increases to $100.5 \%$ of its initial value and decreases to 99.8\% thereof while after one full cycle it again takes its initial value. These are representative values for argon bubbles near the onset of the SL regime; for larger forcing, the exchange processes become even more violent.

\section{F. Organization of the paper}

The paper is organized as follows. In Section II we analyze the bubble stability with respect to shape oscillations. We then give as necessary criterion for light emission energy focusing in the bubble, either through a shock or through acoustic resonance (Section III). Section IV constitutes the main part of the paper. We calculate the diffusive instability with Fyrillas and Szeri's ${ }^{37}$ and Löfstedt et al.'s ${ }^{7}$ adiabatic approximation. The main results are phase diagrams in the $c_{\infty}-P_{a}$, the $R_{0}-P_{a}$, and the $R_{0}-c_{\infty}$ parameter spaces. Only in a very small parameter domain does stable SL occur. To analyze unstable SL we calculate growth rates and compare them to Putterman's measurements of diffusively unstable SL argon bubbles. ${ }^{5}$ Concentration profiles from a full numerical solution of the advection diffusion PDE are presented in Section V, where we also check the validity of the adiabatic approximation by comparison with full numerical solutions of diffusive dynamics. We find experimentally undetectable discrepancies which vanish in the Schmidt number Sc $\rightarrow \infty$ limit. Section VI presents conclusions.

\section{SHAPE STABILITY}

For sonoluminescence to occur and for the bubble to remain oscillating for billions of cycles, the bubble must be stable to shape oscillations. First, following the pioneering work of Plesset, ${ }^{12}$ Strube, ${ }^{15}$ and Prosperetti, ${ }^{16}$ we derive equations for the deviations of the bubble from a spherical shape, and then proceed to analyze them.

\section{A. Dynamical equations}

We focus on the stability of the radial solution $R(t)$. Consider a small distortion of the spherical interface $R(t)$,

$$
R(t)+a_{n}(t) Y_{n}(\theta, \phi),
$$

where $Y_{n}$ is a spherical harmonic of degree $n$. The goal is to determine the dynamics $a_{n}(t)$ for each mode. Plesset's ${ }^{12}$ derivation follows the same spirit as the derivation of the Rayleigh-Plesset equation. The potential flow outside the bubble is constructed to satisfy the boundary condition that the velocity at the bubble wall is $\dot{R}+\dot{a}_{n} Y_{n}$. This potential is then used in Bernoulli's law to determine the pressure in the liquid at the bubble wall. Applying the pressure jump condition across the interface yields the Rayleigh-Plesset equation for $R(t)$ as well as a dynamical equation for the distortion amplitude $a_{n}(t)$,

$$
\ddot{a}_{n}+\frac{3 \dot{R}}{R} \dot{a}_{n}-\left[(n-1) \frac{\ddot{R}}{R}-\frac{\beta_{n} \sigma}{\rho_{w} R^{3}}\right] a_{n}=0,
$$

where $\beta_{n}=(n-1)(n+1)(n+2)$. However, viscous effects have been neglected in Plesset's derivation.

Viscosity was later taken into account by Prosperetti. ${ }^{16}$ The intrinsic difficulty in its consideration is that viscous stresses produce vorticity in the neighborhood of the bubble wall. $^{44}$ In principle, vorticity spreads both by convective and by diffusive processes all over the fluid and the problem becomes nonlocal. However, for small viscosity the generated vorticity will be more or less localized and we can introduce a bubble boundary layer approximation of the nonlocal equations which we do in the next subsection.

Here, we give the dynamics of the nonlocal problem, closely following Prosperetti. ${ }^{16}$ It is advantageous to decompose the vorticity field in the fluid in a poloidal and a toroidal part, which are conveniently represented by scalar fields $S(r, t)$ and $T(r, t)$, respectively, 


$$
\begin{aligned}
\boldsymbol{\omega}= & \nabla \times \nabla \times\left[S(r, t) Y_{n}^{m}(\theta, \phi) \mathbf{e}_{\mathbf{r}}\right] \\
& +\nabla \times\left[T(r, t) Y_{n}^{m}(\theta, \phi) \mathbf{e}_{\mathbf{r}}\right] .
\end{aligned}
$$

Only the latter, $T(r, t)$, contributes to the long term dynamics of the bubble. Its dynamics are given by the PDE

$$
\partial_{t} T(r, t)+R^{2} \dot{R} \partial_{r}\left(\frac{1}{r^{2}} T\right)=\nu \partial_{r}^{2} T-\frac{\nu n(n+1)}{r^{2}} T,
$$

by the nonlocal boundary condition at $R(t)$,

$$
\begin{aligned}
& T(R, t)+2 R^{n-1} \int_{R}^{\infty} s^{-n} T(s, t) d s \\
& \quad=\frac{2}{n+1}\left[(n+2) \dot{a}_{n}-(n-1) \frac{a_{n}}{R} \dot{R}\right],
\end{aligned}
$$

and by the boundary condition at infinity, $T(\infty, t)=0$. At $t=0$ the fluid is assumed to be at rest. The collapse $\dot{R}(t)$ of the bubble transports vorticity $\boldsymbol{\omega}$ into the fluid, see Eq. (6).

Once created, the vorticity acts back on the dynamics of $a_{n}(t)$. These indirect viscous corrections together with the direct ones modify Eq. (4) to yield

$$
\begin{array}{r}
\ddot{a}_{n}+B_{n}(t) \dot{a}_{n}-A_{n}(t) a_{n}+n(n+1)(n+2) \frac{\nu}{R^{2}} T(R, t) \\
-n(n+1) \frac{\dot{R}}{R^{2}} \int_{R}^{\infty}\left[1-\left(\frac{R}{s}\right)^{3}\right]\left(\frac{R}{s}\right)^{n} T(s, t) d s=0
\end{array}
$$

with

$$
\begin{aligned}
& A_{n}(t)=(n-1) \frac{\ddot{R}}{R}-\frac{\beta_{n} \sigma}{\rho_{w} R^{3}}+2 \beta_{n} \nu \frac{\dot{R}}{R^{3}}, \\
& B_{n}(t)=\frac{3 \dot{R}}{R}-2 \beta_{n} \frac{\nu}{R^{2}} .
\end{aligned}
$$

\section{B. Boundary layer approximation}

For an exact stability analysis the coupled Eqs. (6)-(8) together with the RP Eq. (2) must be solved. However, considerable vorticity is only to be expected in a small boundary layer of thickness $\delta$ around the bubble.

Within this boundary layer approximation the space integrals in (7) and (8) can be approximated by the (integrand at $R) \times \delta$. The integral in (7) thus is $\approx R^{-n} T(R, t) \delta$ while the one in (8) vanishes. We obtain

$$
\ddot{a}_{n}+B_{n}(t) \dot{a}_{n}-A_{n}(t) a_{n}=0
$$

with

$$
\begin{aligned}
A_{n}(t)= & (n-1) \frac{\ddot{R}}{R}-\frac{\beta_{n} \sigma}{\rho_{w} R^{3}}-\frac{2 \nu \dot{R}}{R^{3}}\left[-\beta_{n}+n(n-1)\right. \\
& \left.\times(n+2) \frac{1}{1+2 \delta / R}\right] \\
B_{n}(t)= & \frac{3 \dot{R}}{R}+\frac{2 \nu}{R^{2}}\left[-\beta_{n}+\frac{n(n+2)^{2}}{1+2 \delta / R}\right] .
\end{aligned}
$$

The viscous contribution to $A_{n}(t)$ is not important and only causes a tiny shift, as the ratio between the third and the second term of the rhs in (12) is typically $\nu \rho_{w} R_{0} \omega / \sigma \sim 10^{-2}$. However, in (13) it introduces a damping rate

$$
\xi_{n}(t)=\frac{2 \nu}{R^{2}}\left[-\beta_{n}+\frac{n(n+2)^{2}}{1+2 \delta / R}\right],
$$

acting on the shape oscillations. That only the second term in (13) contributes to the damping rate of the oscillator can formally best be seen after the substitution ${ }^{12} b_{n}(t)$ $\propto(R(t))^{3 / 2} a_{n}(t)$. Physically this is not surprising, as the first term is a mere consequence of spherical geometry. Two physical effects contribute to the damping rate: (i) Stabilizing, local damping by viscous dissipation. If this process is dominant, the viscous boundary layer around the bubble vanishes $(\delta=0)$ and the damping rate becomes $\xi_{n}(t)=2 \nu(n+2)(2 n+1) / R^{2}>0$. (ii) The movement of eddies around the bubble, generated by the shape oscillations itself. With increasing boundary layer thickness $\delta$ (i.e., with increasing viscosity) this destabilizing effect becomes stronger.

How does one approximate the thickness $\delta$ of the boundary layer when we have non-vanishing vorticity? For large bubbles $R \gg \delta$ it is set by the diffusive length scale $\sqrt{\nu / \omega}=2.5 \mu \mathrm{m}$ in Eq. (6). ${ }^{45}$ As a typical frequency scale we choose the forcing frequency $\omega$. Higher frequencies are of course also present in the RP dynamics, but a Fourier analysis of the $R(t)$ signal shows that the forcing frequency is dominant. Because of the angular contribution to the dissipation (the second term on the rhs of Eq. (6)) we also expect a slight dependence on the spherical mode $n$ which we neglect here.

For small bubbles $R \ll \delta$ we do not expect the boundary layer around the bubble to be larger than the bubble itself. We thus have to introduce a cutoff. ${ }^{46}$ We choose

$$
\delta=\min \left(\sqrt{\frac{\nu}{\omega}}, \frac{R}{2 n}\right) .
$$

The $n$-dependence of the cutoff can be understood from the quasi-static limit which holds for small bubbles, as for small bubbles the bubble dynamics is strongly damped by viscosity and $R(t)$ does not change much. In this quasi-static limit the lhs of (6) vanishes and $T(r)=T(R)(r / R)^{-n}$ is the static solution. It decays to half its boundary value $T(R)$ at $r=2^{1 / n} R$. Thus $\delta=R\left(2^{1 / n}-1\right) \approx R \ln 2 / n \approx R /(2 n)$ as in (15). More precisely, for $T(r)=T(R)(r / R)^{-n}$ we can calculate $T(R)$ from Eq. (7), and obtain essentially the same $\delta$. The exact values of our results depend on details of the cutoff (15). However, the general features of the solution are invariant.

With the approximation (15) we can Taylor-expand (12) and (13) and finally obtain as approximate dynamical equation for $a_{n}(t) \mathrm{Eq}$. (11) with ${ }^{17}$ 

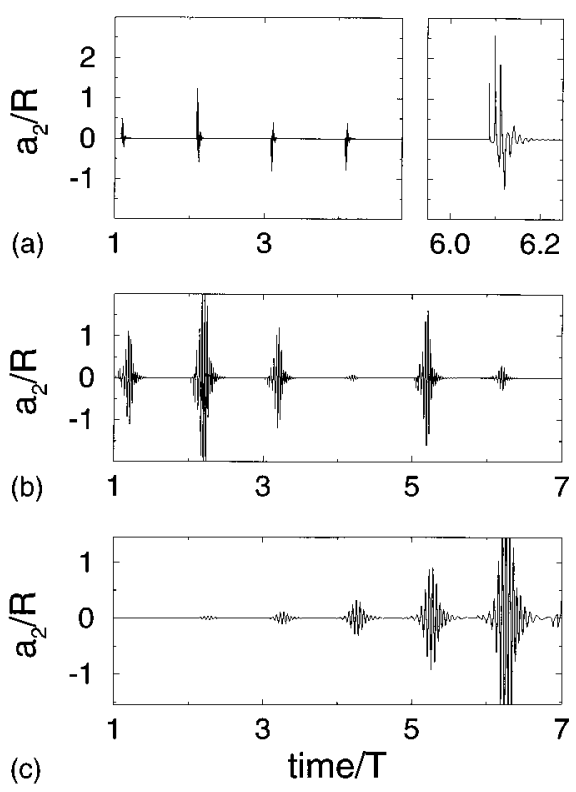

FIG. 3. Time development of the normalized distortion amplitude $a_{2}(t) / R(t)$ for (a) a Rayleigh-Taylor unstable, parametrically stable bubble $\left(R_{0}=2.5 \mu \mathrm{m}, P_{a}=1.5 \mathrm{~atm}\right)$, (b) an afterbounce unstable, parametrically stable bubble $\left(R_{0}=4.0 \mu \mathrm{m}, P_{a}=1.3 \mathrm{~atm}\right)$ and (c) a Rayleigh-Taylor stable, parametrically unstable bubble $\left(R_{0}=5.2 \mu \mathrm{m}, P_{a}=1.0 \mathrm{~atm}\right)$. In (a) we also show $a_{2} / R$ in a blow up of the time scale to demonstrate that the typical time scale of the Rayleigh-Taylor instability is nanoseconds. The typical time scales of afterbounce (b) and parametric instability (c) is in the microsecond, and millisecond range, respectively.

$$
\begin{aligned}
A_{n}(t)= & (n-1) \frac{\ddot{R}}{R}-\frac{\beta_{n} \sigma}{\rho_{w} R^{3}}-\frac{2 \nu \dot{R}}{R^{3}}[(n-1)(n+2) \\
& \left.+2 n(n+2)(n-1) \frac{\delta}{R}\right], \\
B_{n}(t)= & \frac{3 \dot{R}}{R}+\frac{2 \nu}{R^{2}}\left[(n+2)(2 n+1)-2 n(n+2)^{2} \frac{\delta}{R}\right] .
\end{aligned}
$$

Our results are based on these equations.

\section{Rayleigh-Taylor, afterbounce, and parametric instabilities}

Three types of shape instabilities are hidden inside these equations. We call them the Rayleigh-Taylor instability, the afterbounce instability, and parametric instability, for reasons which will become clear later. They are distinguished by the widely different time scales over which they act. The transition between these instabilities is often gradual rather than abrupt. Nevertheless, we think that our classification is physically important as the difference in their time scales results in a difference of the typical velocities of the bubble fragments after the shape instability has destroyed the bubble. We estimate this velocity as typical length scale $\sim 1 \mu \mathrm{m}$ of a collapsed bubble divided by the typical time scale of the pinch off.

The goal of this subsection is to find criteria for the occurrence of the three shape instabilities. Their nature becomes clear from Fig. 3, where we show the dynamics of $a_{2}(t)$ (which is the most unstable spherical mode in the parameter range discussed in this paper, so we restrict ourselves to it) for the three different regimes of instability. We normalize $a_{2}(t)$ to the current bubble radius $R(t)$ to get a measure of bubble distortion. The upper part displays the dynamics of the distortion amplitude $a_{2}(t)$, normalized to $R(t)$, in a Rayleigh-Taylor unstable, parametrically stable case, obtained from a numerical solution of Eqs. (2), (11), (16), and (17), with addition of small amplitude noise. We chose Gaussian distributed microscopic fluctuations with a typical size of $a_{2} \sim 1 \mathrm{~nm}$ which is not visible on the scale shown in Figure 3. The middle part shows the dynamics of the distortion in the regime of the afterbounce instability, and the lower part typifies the dynamics of a parametrically unstable, Rayleigh-Taylor stable bubble. Clearly, very different time scales are responsible for the distortion of the spherical shape of the bubble. Of course there are regimes in the $R_{0}-P_{a}$ parameter space where the bubble is stable (or unstable) towards two or even all three instabilities.

First we focus on the Rayleigh-Taylor instability, occurring near the minimum bubble radius when the gas accelerates into the fluid. The strongest destabilization occurs just when the bubble radius reaches its minimum. The acceleration of gas towards the fluid during this time is enormous, motivating the name Rayleigh-Taylor instability. Closer analysis of this section of bubble movement ${ }^{47}$ reveals that the time scale of the Rayleigh-Taylor instability can be estimated by the expansion time scale of $R(t)$ just after the collapse which is $t_{\mathrm{RT}} \sim h / c_{w}$ with the van der Waals hard core radius $h=R_{0} / 8.86$. Thus, $t_{\mathrm{RT}} \sim 10^{-9}-10^{-10} \mathrm{~s}$, which is confirmed by the numerical results. In order to take into account microscopic fluctuations we added a random displacement of size $\sim 0.1 \mathrm{~nm}$ to the distortion $a_{2}(t)$ after each integration time step.

For lower $P_{a}$ the destabilization during the violent bubble collapse may not be strong enough to immediately overwhelm the bubble. But as seen from Fig. 3 further periods of destabilization occur during the afterbounces. As pointed out above, in the afterbounce regime $R(t)$ oscillates on the bubble's intrinsic time scale $t_{I} \sim \sqrt{\rho_{w} R_{0}^{2} / P_{0}} \sim 1 \mu \mathrm{s}$. This is too fast for viscous effects to smooth out the shape distortions, so after a couple of afterbounces the bubble may be overwhelmed. This type of instability shows features of parametric instability, however, the afterbounces are not strictly periodic. As an approximate criterion for this afterbounce instability we give that microscopic fluctuations can overwhelm the bubble within one period $T$,

$$
\max _{\left\{t^{\prime} \mid t<t^{\prime}<t+T\right\}}\left(\frac{\left|a_{2}\left(t^{\prime}\right)\right|}{R\left(t^{\prime}\right)}\right) \gtrsim 1 .
$$

Our results only weakly depend on the exact strength of the microscopic fluctuations.

The transition between Rayleigh-Taylor and afterbounce instabilities is illustrated in Fig. 4. The $a_{2} / R$ time series shows violent behavior at the main bubble collapse and the first afterbounces. Then, the behavior of $a_{2}(t) / R(t)$ becomes oscillatory and locks into the periodicity of the $R(t)$ afterbounces with twice their period (the same is true 


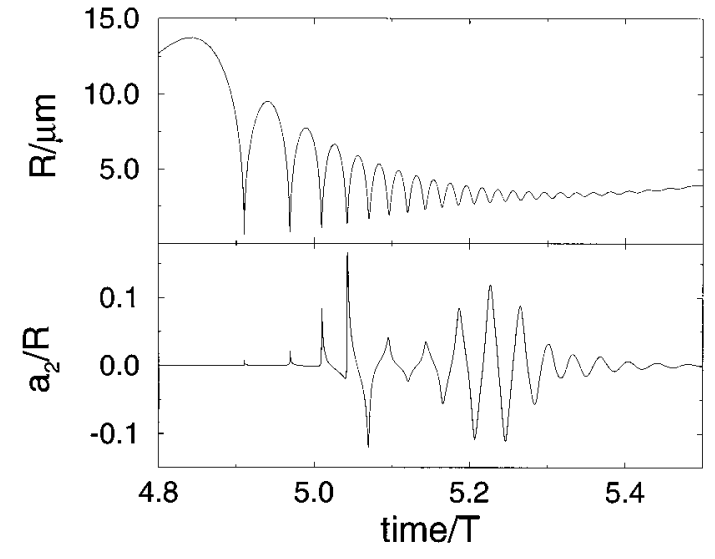

FIG. 4. Time development of the bubble radius $R(t)$ (upper part) and distortion amplitude $a_{2}(t)$ (lower part) for a $R_{0}=4.4 \mu \mathrm{m}$ bubble driven at $P_{a}=1.1 \mathrm{~atm}$. Note the transition from Rayleigh-Taylor (time scale ns) to afterbounce perturbations (time scale $\mu \mathrm{s}$ ) during the afterbounce part of the bubble dynamics. It is also seen that the dynamics of the distortion $a_{2}(t)$ has half the frequency of its forcing bubble dynamics $R(t)$ as typical for an instability of the Mathieu type.

for $a_{2}(t)$ itself). This is to be expected from a Mathieu type instability, which is most effective for a driving with twice the intrinsic frequency of the driven equation.

Figure 5 depicts a phase diagram of shape instabilities as a function of the ambient bubble radius $R_{0}$ and the forcing pressure amplitude $P_{a}$ for $n=2$. The dashed line gives the combined stability threshold for Rayleigh-Taylor and afterbounce instabilities (the "fast" instabilities with time scales $\ll T)$. The global features of the phase diagram are easily understood. Small bubbles are more stable than large ones thanks to viscosity, as the second term in (17) becomes dominant for small radii $R$. Evidently, weakly forced bubbles are more stable than strongly forced ones.

A pure parametric shape instability acts on the much longer time scale of the forcing $T \approx 38 \mu \mathrm{s}$. It corresponds to a net growth of a nonspherical perturbation over one oscillation period, so that after many periods perturbations overwhelm the bubble. The time scale of the parametric instabil-

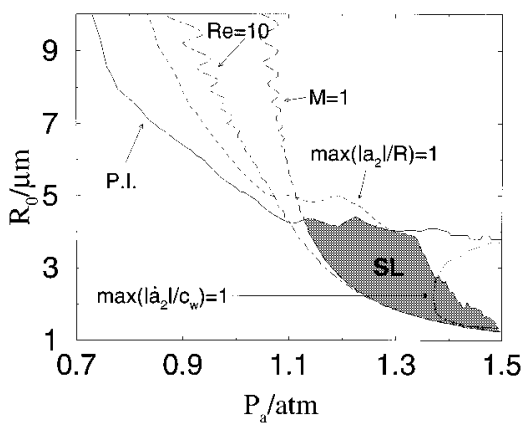

FIG. 5. Borderline of the parametric instability (solid), the afterbounce instability according to criterion (18) (short dashed), and the $M=1$ criterion (22) for a supersonic bubble collapse (long dashed). The $\mathrm{Re}=10$ - criterion (24) for the persistence of a shock (dot-dashed) is found to be less stringent than the $M=1$ criterion. Also shown is the perturbation velocity threshold (21) (dotted). In case of unstable SL, to the right of this curve the bubble is thrown out of the trap as the pinch off of microbubbles is too violent. The region where SL is possible is shaded. ity $t_{\mathrm{PI}}$ is thus many forcing periods $T=2 \pi / \omega$. However, as the finally resulting pinchoff occurs during afterbounces, its time scale is the same as for the afterbounce instability, i.e., the intrinsic time scale of the bubble motion $t_{I} \sim 1 \mu \mathrm{s}$.

In the relevant parameter regime for the parametric instability $R(t)$ and thus also $A_{n}(t)$ and $B_{n}(t)$ are strictly periodic in time with frequency $1 / T$. Thus Eq. (11) is an ODE of Hill's type and the parametric instability can be rigorously analyzed. It occurs whenever the magnitude of the maximal eigenvalue of the Floquet transition matrix $F_{n}(T)$ of Eq. (11) is larger than one. The Floquet transition matrix $F_{n}(T)$ is defined by

$$
\left(\begin{array}{l}
a_{n}(T) \\
\dot{a}_{n}(T)
\end{array}\right)=F_{n}(T)\left(\begin{array}{l}
a_{n}(0) \\
\dot{a}_{n}(0)
\end{array}\right) .
$$

By numerically computing the eigenvalues of the Floquet transition matrix we mapped out the phase diagram of stability. Figure 5 shows the stable and unstable domains in the $R_{0}-P_{a}$ parameter space.

In the SL parameter range of $P_{a} \approx 1.2$ to $1.5 \mathrm{~atm}$ the bubble becomes parametrically unstable at about $R_{0}^{\mathrm{PI}} \approx 4-5 \mu \mathrm{m}$. This number is not to be understood as a prediction of the exact value, as within our approximations we can only predict the order of magnitude and trends.

For smaller $P_{a}$ the threshold for instability $R_{0}^{\mathrm{PI}}$ does depend on the forcing pressure. We discussed phase diagrams in those regimes in Ref. 17 and also showed that in the small forcing limit Eq. (11) reduces to a Mathieu equation.

That $R_{0}^{\mathrm{PI}}$ does not significantly depend on $P_{a}$ for large $P_{a}$ can be understood from the dynamics of $R(t)$ and $a_{n}(t)$ and from Eq. (17). If $R(t)$ is small, the second term in (17) dominates and stabilizes $a_{n}(t)$. For small $P_{a}$ the minimal radius $R_{\min }=\min _{t}(R(t))$ still decreases with increasing $P_{a}$. But after the van der Waals hard core radius $h=R_{0} / 8.86$ has once been reached for large enough $P_{a}, R_{\min }$ becomes $P_{a}$ independent. $^{47}$

All calculations have been performed for the viscosity of water $\nu=10^{-6} \mathrm{~m}^{2} / \mathrm{s}$. Of course $R_{0}^{\mathrm{PI}}$ and the other thresholds strongly depend on $\nu$, e.g., for a viscosity five times that of water we have $R_{0}^{\mathrm{PI}} \approx 10 \mu \mathrm{m}$, but we won't discuss this dependence here.

\section{After the shape instabilities}

All types of shape instabilities result in the pinching off of microbubbles. In other experimental configurations such as the Faraday experiment, ${ }^{24}$ parametric instability can saturate at a finite amplitude. This is also possible for larger bubbles which are driven with small forcing pressure $P_{a}<1$ atm. ${ }^{1,33}$ However, we believe the nonlinear saturation is unlikely in the present experiment because the bubble size changes by two orders of magnitude during a single oscillation period. Saturation would require that the amplitude $a_{n}$ is much smaller than the minimum radius $R_{\text {min }}$; however, since the bubble spends most of the cycle with $R \gg R_{\min }$, the nonlinearities mainly act at larger radii. 
The major question, therefore, is what happens after the pinch off of a microbubble. Will the bubble remain trapped at the antinode of the pressure field, or will it escape from the system?

The force holding the bubble in the trap is the so-called Bjerknes force given by ${ }^{33,48}$

$$
\mathbf{F}=-\frac{4}{3} \pi R^{3} \nabla P .
$$

Both $R(t)$ and $\boldsymbol{\nabla} P(t)$ are oscillating with time. The combination of both will lead to an effective, period averaged force $\langle\mathbf{F}\rangle$ which pushes bubbles smaller than the resonance radius $(\sim 100 \mu \mathrm{m}$ here $)$ to the pressure antinode..$^{33}$ The bubbles are thus trapped.

During the collapse the forcing pressure is positive and the force (20) repulsive. If microbubbles pinch off at this instant, will they and the recoiled bubble be fast enough to escape from the node before the force becomes attractive again? If so, they must travel a quarter of the acoustic wavelength $c_{w} T / 4$ in time $T / 2$, thus their velocity must be of order $\sim c_{w} \sim 10^{3} \mathrm{~m} / \mathrm{s}$. Assuming a typical length scale of $R_{0} \sim 1 \mu \mathrm{m}$ for the microbubble and the remainder, we obtain as a critical time scale of the collapse $10^{-9} \mathrm{~s}$ : If the collapse is faster, the bubble cannot survive in the trap, if it is slower, it is likely to survive as a smaller bubble. From the estimates of the relevant collapse time scale in the last subsection we conclude that the bubble will survive the afterbounce and the parametric instability where the pinch off occurs on the intrinsic time scale $t_{I} \sim 1 \mu \mathrm{s}$. We assume that the pinched off microbubble from those shape instabilities will dissolve by diffusion (see below), but the remainder of the bubble may survive with some probability (i.e., if it is large enough) and grow by rectified diffusion. Another possibility is that the bubble fragments may remerge. Note that the recoil of the bubble on fragmentation is the origin of the jiggling or dancing of the bubble as we will discuss later.

The bubble fragments can, however, also be ejected from the trap after a RT instability $\left(t_{\mathrm{RT}} \sim 10^{-9} \mathrm{~s}\right)$. To get a more stringent criterion for ejection, we computed the maximal velocity $\left|\dot{R}+\dot{a}_{2}\right|$. For a bubble split apart by shape instabilities, this will also give the typical fragment velocity. The outward velocity is dominated by the $\dot{a}_{2}$ term; $\dot{R}$ does not exceed $0.1 c_{w} \cdot{ }^{47}$ Therefore, we assume that the fragments will escape if

$$
\max _{\left\{t^{\prime} \mid t<t^{\prime}<t+T\right\}}\left(\frac{\left|\dot{a}_{2}\left(t^{\prime}\right)\right|}{c_{w}}\right) \geqslant 1 .
$$

Figure 6 depicts the lhs of this inequality as a function of $P_{a}$ and $R_{0}$. The perturbation speed exceeds the sound speed in two clearly distinct regions of parameter space: at large radii, where the parametric instability leads to high velocity, and at small radii and high pressures, where $c_{w}$ is reached during Rayleigh-Taylor instability. Only the latter region will determine the escape of the bubble, because parametrically unstable bubbles already shed microbubbles long before the high velocities associated with the RT instability discussed here are reached (these high velocities would only occur for $\left|a_{2}\right| \gg R$ ). We extract a $\left|\dot{a}_{2}\right|=c_{w}$ isoline from this graph and add it to the phase diagram in Fig. 5 (dotted line).

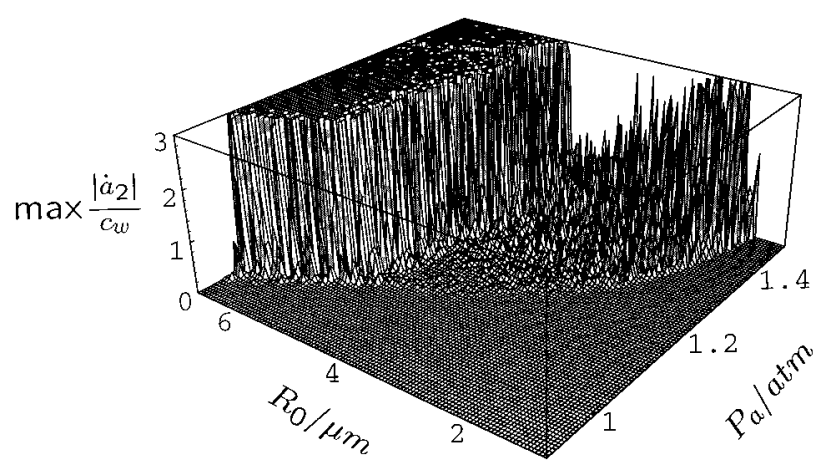

FIG. 6. Maximum perturbation velocity according to (21) as a function of $P_{a}$ and $R_{0}$. This calculation was done with an $a_{2}$ noise amplitude of $1 \mathrm{~nm}$. Note the two distinct regions of the parametric instability (left) with its rapid onset and the Rayleigh-Taylor instability (right, high pressures and small $R_{0}$ ). In the latter region the bubble is thrown out of the trap when the pinch off occurs. It corresponds to the region right of the dotted line in Fig. 5.

In accord with the estimate for $t_{\mathrm{RT}}$ in the preceding section, bubbles with smaller $R_{0}$ have faster instability time scales and thus higher values of $\left|\dot{a}_{2}\right|$. However, for very small $R_{0}$, the bubble dynamics is stabilized by surface tension, and the perturbation velocity drops again. The exact position of this line is not meant to be quantitative, because it is extracted from dynamical data for bubble velocities where the RP equation is not a good approximation to bubble dynamics any more.

\section{ENERGY FOCUSING MECHANISM}

Although this paper is primarily concerned with stability constraints on a bubble obeying the Rayleigh-Plesset equation, in order to relate the calculations to the sonoluminescence experiments it is necessary to adopt an onset criterion for the light emission. The light production is triggered by a hydrodynamic energy focusing mechanism, which concentrates the input energy enough so that light is produced. The mechanism through which the focused energy produces light is outside the scope of this paper; many suggestions such as bremsstrahlung or blackbody radiation are present in literature. $^{22,6}$

Two theories of hydrodynamic energy focusing have been proposed: The original theory was that during the collapse of the bubble shocks detach from the gas-water interface and focus to the bubble's center. ${ }^{21,22}$ Strictly speaking the shock does not "detach" from the wall but forms slightly afterwards. The motion of a focusing shock is described by Guderley's similarity solution to the hydrodynamic equations, ${ }^{49,24}$ which dictates that the temperature at the shock diverges as $T \propto R_{s}^{-p}$ where $R_{s}$ is the distance of the shock from the origin and $p \sim 1$ is an irrational scaling exponent. The amount of energy focusing in this theory is determined by the minimum distance $R_{s, \min }$ to which the shock approaches the origin.

The second theory of energy focusing ${ }^{23}$ posits that the bubble is an "acoustic resonator," and that acoustic energy builds up in the bubble over many oscillation periods. Within this picture the amount of energy focusing is set by the total stored energy in the bubble. 
The crucial issue for the present paper is the onset criterion for how strongly the bubble must be forced for significant energy focusing to occur. Energy can only be transferred from the liquid to the gas if the time scale of motion of the bubble wall is of the order of the time scale of acoustic modes in the gas. The forcing time scale is $R /|\dot{R}|$ and the intrinsic acoustic resonator time scale is $R / c_{\text {gas }}$, where $c_{\text {gas }}$ is the speed of sound in gas. Thus the inward bubble wall velocity $|\dot{R}|$ must be (at least) of order of $c_{\text {gas }}$, or introducing the Mach number $M$,

$$
M=\frac{-\dot{R}}{c_{\text {gas }}} \gtrsim 1 .
$$

From the above discussion of the time scales in the RP equation it follows that all time scales between $R /|\dot{R}|$ and $T$ are present in the $R(t)$ dynamics; thus (22) is the correct criterion for energy focusing. The speed of sound near the bubble wall is

$$
c_{\mathrm{gas}}^{2}=\gamma \frac{p}{\rho} \frac{R^{3}}{R^{3}-h^{3}} .
$$

As in Eq. (3), we have assumed that the gas near the bubble wall can be parametrized with an isothermal van der Waals equation; an approximation which clearly breaks down during compression in the center of the bubble.

For large enough forcing the energy focusing criterion (22) is fulfilled once per cycle, namely shortly before the bubble achieves its minimum radius which in the relevant $P_{a}$ domain is very close to the hard core radius $h$. This is where the light pulse is emitted ${ }^{1,2,36}$ which gives support to the criterion (22). In Fig. 5 we plotted the threshold for the $M=1$ criterion in the $P_{a}-R_{0}$ parameter domain.

Let us check two further conditions which should be fulfilled within the shock wave theory. First, another requirement besides (22) is that the shock must persist. Dissipative mechanisms inside the bubble must therefore be weak. A measure for the relative strength of inertial and dissipative mechanisms is the Reynolds number Re. Viscous effects dominate for small Re. As crossover for nonlinear effects such as shocks to take over we take ${ }^{24}$

$$
\mathrm{Re}=\frac{R|\dot{R}|}{\nu_{\text {gas }}} \gtrsim 10 .
$$

The kinematic viscosity of argon is $\nu_{\text {gas }}=11 \times 10^{-6} \mathrm{~m}^{2} / \mathrm{s}$. We neglect its temperature dependence. In the center of the bubble this is a poor approximation and $\nu_{\text {gas }}$ will be lower. The $\operatorname{Re}>10$ criterion will then be fulfilled earlier. However, (22) is the more stringent criterion anyhow as seen from Fig. 5.

Second, we have to compare the thickness of a shock with the bubble's size. The thickness of a shock is of the same order of magnitude as the mean free path $l$ of a gas molecule. ${ }^{24}$ We have $l \sim V /\left(N \sigma_{0}\right)$ where $V$ is the bubble volume, $N$ the number of particles in the bubble, and $\sigma_{0} \sim 10^{-19} \mathrm{~m}^{2}$ the collision cross section of argon atoms. Thus

$$
l=l_{0}\left(\frac{R}{R_{0}}\right)^{3} .
$$

With $l_{0} \sim 10^{-7} \mathrm{~m}$ we obtain $l \sim 10^{-10} \mathrm{~m}$ during the strongest compression, i.e., a very sharply defined shock with a width $\ll R$ in spite of viscosity.

We now come back to Fig. 5. That plot summarizes the criteria we suggest to be necessary for SL to occur: (i) Bubble wall Mach number $M>1$ to ensure energy focusing to reach the high temperatures necessary for SL. (ii) Short time scale shape stability (Rayleigh-Taylor and afterbounce) and (iii) parametric stability. (iv) Finally, the perturbation speed must not exceed $c_{w}$, in order to keep the bubble or its fragments trapped in the sound field.

There is only a small domain in parameter space where the bubble fulfills all four criteria. This domain is shaded in Fig. 5. It is this domain where we expect SL to be possible (within our RP-SL approach). However, up to now no statement on the diffusive stability has been made. We will address this subject in the next sections and find that for low enough gas concentration the bubble in the shaded domain is also diffusively stable.

\section{DIFFUSIVE STABILITY}

Two types of SL in argon bubbles have been observed: ${ }^{5}$ For large argon concentrations $P_{\infty}=200 \mathrm{mmHg}$ or $50 \mathrm{mmHg}$ the SL bubble is diffusively unstable whereas for low concentrations $P_{\infty}=3 \mathrm{mmHg}$ the bubble is diffusively stable and the relative phase of light emission stays constant for hours, see Fig. 11(c) for the experimental result. With the ambient pressure $P_{0}=760 \mathrm{mmHg}=1$ atm these three gas concentrations translate into relative concentrations of $c_{\infty} / c_{0}=0.26,0.06$, and 0.004 , respectively, where $c_{0}=0.061 \mathrm{~kg} / \mathrm{m}^{3}$ is the saturation (mass) concentration of argon in water for room temperature.

In this section we set out to quantitatively understand the difference between the high and low concentration. Our goal is to calculate a phase diagram in the parameter space of the two experimental control parameters concentration $c_{\infty} / c_{0}$ and forcing $P_{a} / P_{0}$. For given concentration and given forcing we will thus be able to predict which of the three phases "diffusively stable SL," "diffusively unstable SL," and "no SL" will be realized. The third parameter, the ambient bubble radius $R_{0}$ is not at the experimenter's disposal but the system will choose $R_{0}$ itself. The ambient radius will follow from our analysis. First, we will present phase diagrams in the ambient radius-concentration and ambient radiusforcing pressure phase space.

We again stress that it is the application of the adiabatic approximation of the diffusive problem ${ }^{37,7}$ which allows for the exploration of the whole 3-D phase space $R_{0}-c_{\infty}-P_{a}$. Strictly speaking, it only holds in the limit of zero diffusion constant $D \rightarrow 0$. However, in the next section we will show that for the physical diffusion constant $D=D_{\mathrm{Ar}}=2$ $\times 10^{-9} \mathrm{~m}^{2} / \mathrm{s}$ the deviations between the exact solution and the adiabatic approximation are tiny, so that we can apply it here. 


\section{A. Formulation of the diffusive problem}

Assuming spherical symmetry, the mass concentration of gas $c(r, t)$ dissolved in the liquid at distance $r>R(t)$ from the center of the bubble obeys the advection diffusion equation

$$
r^{2} \partial_{t} c+R^{2} \dot{R} \partial_{r} c=D \partial_{r}\left(r^{2} \partial_{r} c\right) .
$$

As a boundary condition at the bubble wall we assume Henry's law

$$
c(R, t)=c_{0} p(R, t) / P_{0} .
$$

The concentration at $r \rightarrow \infty$ is given by $c_{\infty}$,

$$
c(\infty, t)=c_{\infty} .
$$

The concentration gradient at the moving boundary gives the mass loss/gain of the bubble

$$
\dot{m}=\left.4 \pi R^{2} D \partial_{r} c\right|_{R(t)} \text {. }
$$

The bubble is driven by the RP Eq. (2). Together with the initial conditions $R(t=0)=R_{0}, \dot{R}(t=0)=0$ and $c(r, t=0)$ $=c_{\infty}$ this set of equations completely defines the problem.

With the transformation to the Lagrangian coordinate ${ }^{35}$

$$
h(r, t)=\frac{1}{3}\left(r^{3}-R^{3}(t)\right)
$$

the advection diffusion PDE (26) simplifies to the diffusive equation

$$
\partial_{t} c(h, t)=D \partial_{h}\left[\left(3 h+R^{3}(t)\right)^{4 / 3} \partial_{h} c(h, t)\right] .
$$

Equation (31) can still not be solved analytically. A numerical treatment of this equation is sketched in Appendix A.

\section{B. Adiabatic approximation}

The main idea of Fyrillas and Szeri ${ }^{37}$ and Löfstedt $e t$ al. ${ }^{7}$ is to treat the diffusive PDE by the method of separation of time scales. ${ }^{50}$ They split the concentration field in an oscillatory part $c_{\text {osc }}(r, t)$ changing on the (fast) time scale $T$ of the driving field and a smooth part $c_{\text {smo }}(r, t)$ changing on a slow diffusive time scale $\tau_{D} \gg T$,

$$
c(r, t)=c_{\text {osc }}(r, t)+c_{\text {smo }}(r, t) .
$$

This approach can be thought of as having introduced an "adiabatic" or slow time $\bar{t}$. The smooth profile $c_{\text {smo }}$ only depends on the adiabatic time, $c_{\mathrm{smo}}(r, \bar{t})$. In the PDE for $c_{\text {smo }}(r, \bar{t})$ the fast time scale $\sim T$ is averaged out. We define $\tau_{D}=R_{0}^{2} / D$ as diffusive time scale. Then the Schmidt number $\mathrm{Sc}=2 \pi \tau_{D} / T$ is a measure of the quality of time scales separation. $\mathrm{Sc} \rightarrow \infty$ or $D \rightarrow 0$ means perfect separation.

It turns out to be useful to introduce weighted time averages,

$$
\langle f(t)\rangle_{t, i}=\frac{\int_{0}^{T} f(t) R^{i}(t) d t}{\int_{0}^{T} R^{i}(t) d t},
$$

which may still depend on the adiabatic time $\bar{t}$. Here, one only needs $\langle\cdot\rangle_{t, 0}$ and $\langle\cdot\rangle_{t, 4}{ }^{7,37}$

The main result of Ref. 37 is that in the asymptotic limit $\bar{t} \rightarrow \infty$ the smooth profile $c_{\text {smo }}(h, \bar{t})$ converges to

$$
\begin{aligned}
\bar{c}_{\mathrm{smo}}(h)= & c_{\infty}+\left[c_{0} \frac{\langle p(t)\rangle_{t, 4}}{P_{0}}-c_{\infty}\right] \\
& \times\left\{1-\frac{\int_{0}^{h} \frac{d h^{\prime}}{\left\langle\left(3 h^{\prime}+R^{3}(t)\right)^{4 / 3}\right\rangle_{t, 0}}}{\int_{0}^{\infty} \frac{d h^{\prime}}{\left\langle\left(3 h^{\prime}+R^{3}(t)\right)^{4 / 3}\right\rangle_{t, 0}}}\right\} .
\end{aligned}
$$

From (34) the adiabatic growth of the bubble can be calculated as

$$
\frac{d}{d \bar{t}} R_{0}(\bar{t})=\frac{D c_{0}}{\rho_{0} R_{0}^{2}(\bar{t})} \frac{\left[\frac{c_{\infty}}{c_{0}}-\frac{\langle p(t)\rangle_{t, 4}(\bar{t})}{P_{0}}\right]}{\int_{0}^{\infty} \frac{d h^{\prime}}{\left\langle\left(3 h^{\prime}+R^{3}(t)\right)^{4 / 3}\right\rangle_{t, 0}}} .
$$

The determination of the adiabatic growth rate has thus been reduced to solving the RP ODE (2) for $R(t)$, calculating time averages $\langle\cdot\rangle_{t, i}$ of functions of $R(t)$, and the solution of a space integral. We thus understand the adiabatic approximation as being in the spirit of the RP approach.

\section{Equilibrium points}

We now apply Eq. (35) to the acoustically driven argon bubble. ${ }^{5}$ Let us disregard the shape instabilities discussed in Section II for the time being.

The ambient bubble radius is in equilibrium (within the adiabatic approximation), if

$$
\frac{c_{\infty}}{c_{0}}=\frac{\langle p(t)\rangle_{t, 4}}{P_{0}} .
$$

Note that within the adiabatic approximation the condition does not depend on the diffusion coefficient $D$. However, the smaller the $D$, the better the adiabatic approximation holds. The equilibrium is stable, if

$$
\beta=\frac{d\langle p(t)\rangle_{t, 4}}{d R_{0}}
$$

is positive. Scaling laws for $\langle p\rangle_{t, 4}$ and $\beta$ are discussed in the next subsection and in Ref. 47; here we concentrate on the physical consequences.

In Fig. 7 we plot $\langle p(t)\rangle_{t, 4}$ as a function of $R_{0}$ for various forcing pressure amplitudes $P_{a}$. We first focus on small $P_{a} \approx 0.8 \mathrm{~atm}$ and high gas concentration of $c_{\infty} / c_{0} \approx 0.7$. There exists an unstable equilibrium at $R_{0}^{e} \approx 6 \mu \mathrm{m}$. Smaller bubbles shrink and finally dissolve, larger bubbles grow by rectified diffusion. For larger $P_{a}$ the average $\langle p(t)\rangle_{t, 4}\left(R_{0}\right)$ behaves quite differently in the small $R_{0}$ regime. It starts to show characteristic wiggles, which can also be seen in $R_{\max }\left(R_{0}\right)$. Here, $R_{\max }$ is the maximal radius over one period, $R_{\max }(t)=\max \left\{R\left(t^{\prime}\right) \mid t \leqslant t^{\prime} \leqslant t+T\right\}$. The origin of the wiggles is a kind of resonance phenomenon in the RP equation and can quantitatively be understood in detail. ${ }^{47}$ Here we only discuss their consequences for the oscillating bubble. They mean that the bubble may stabilize through a tangent bifurcation: Imagine a fixed forcing $P_{a}$ and then decrease $c_{\infty} / c_{0}$. The $c_{\infty} / c_{0}$ line will finally touch $\langle p\rangle_{t, 4} / P_{0}$ at a local maximum and create a pair of stable and unstable fixed 

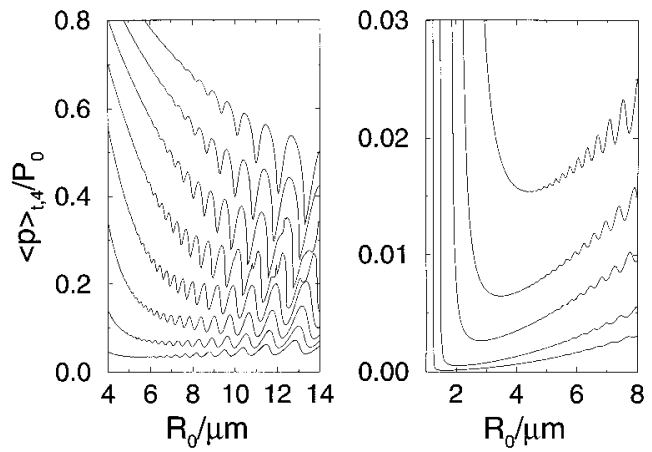

FIG. 7. $\langle p\rangle_{t, 4} / P_{0}$ as a function of the ambient radius $R_{0}$ for small forcing pressure amplitudes $P_{a}=0.8$ atm to $P_{a}=1.15$ atm (left, top to bottom, in steps of $0.05 \mathrm{~atm}$ ) and for large $P_{a}=1.2,1.25,1.3,1.4,1.5 \mathrm{~atm}$ (right, top to bottom). Note the different ordinate scales.

points which will separate for decreasing $c_{\infty} / c_{0}$. This process can repeat many times. The fixed points vanish through inverse tangent bifurcations, i.e., stable and unstable fixed points merge. The full bifurcation diagram is shown in Fig. 8 for $P_{a}=1.1 \mathrm{~atm}$ and for $P_{a}=1.3 \mathrm{~atm}$.

The interpretation of the $R_{0}-c_{\infty}$ phase diagram is as follows. The line signals equilibrium for the given driving pressure $P_{a}$. Again, we denote the equilibrium radius as $R_{0}^{e}$. It is stable if the slope $\partial R_{0}^{e} /\left.\partial c_{\infty}\right|_{P_{a}}$ is positive, and unstable if it is negative. To the left of the line the bubbles shrink, to the right of it they grow by rectified diffusion. The shrinking or growing bubbles can hit a stable fixed point and thus stabilize. The basin of attraction of the stable fixed points is considerably larger for larger forcing $P_{a}$, see Fig. 8 .

A similar looking phase diagram results when fixing $c_{\infty} / c_{0}$ and varying $P_{a}$. In Fig. 9 we show such a bifurcation diagram in the $R_{0}-P_{a}$ parameter space for large concentration $c_{\infty} / c_{0}=0.5$, for lower concentration $c_{\infty} / c_{0}=0.02$, and for very low concentration $c_{\infty} / c_{0}=0.002$. Again, the lines signal equilibrium; stable equilibrium for positive slope $\partial R_{0}^{e} /\left.\partial P_{a}\right|_{c_{\infty}}$, unstable equilibrium for negative slope. To the right of the line we have growth, left of it shrinking. Increasing $P_{a}$ at fixed $c_{\infty}$ again leads to stabilization through a

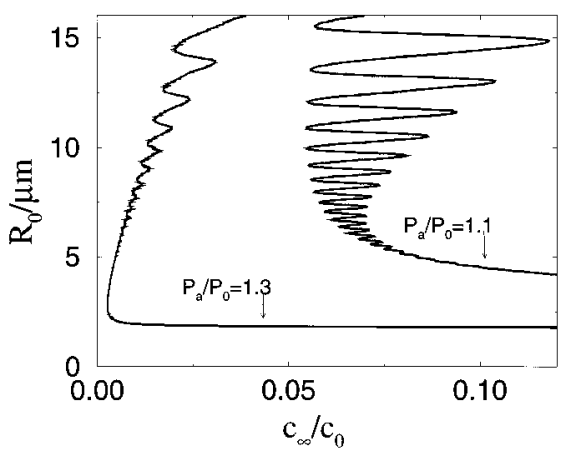

FIG. 8. Bifurcation diagrams in the $R_{0}-c_{\infty}$ parameter plane for a forcing pressure of $P_{a}=1.3 \mathrm{~atm}$ (left) and $P_{a}=1.1 \mathrm{~atm}$ (right). Tangent bifurcations are seen. The regimes with positive slope are stable. To the left of the curves the bubbles shrink, to the right of them they grow.

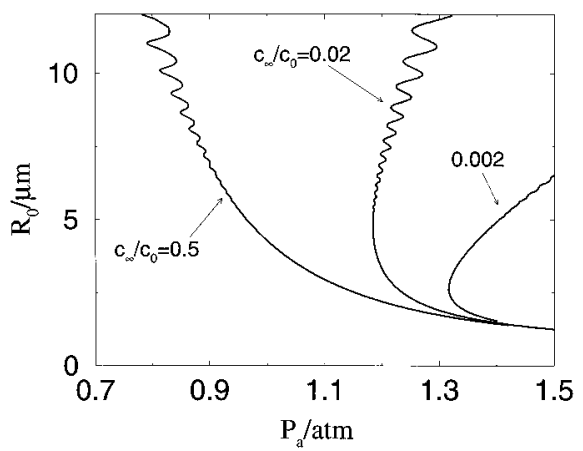

FIG. 9. Bifurcation diagrams in the $R_{0}-P_{a}$ parameter space. Tangent bifurcations are seen. The regimes with positive slope are stable. Gas concentrations are $c_{\infty} / c_{0}=0.002$ (right), $c_{\infty} / c_{0}=0.02$ (middle), and $c_{\infty} / c_{0}=0.5$ (left). To the left of the curves the bubbles shrink and finally dissolve, to the right of them they grow by rectified diffusion.

series of tangent bifurcations and later on destabilization by inverse tangent bifurcations.

From Figs. 8 and 9 we immediately understand why there is no diffusively stable SL for large Ar concentration. ${ }^{42}$ No diffusively stable bubble radii exist in the large $P_{a}$-small $R_{0}$ parameter regime where the energy focusing condition for SL (22) is fulfilled. Note that there are stable equilibria, but for smaller $P_{a}$ and large $R_{0}$. Note also that the basin of attraction of these stable equilibria is tiny.

For small concentration $c_{\infty} / c_{0}$ the situation is quite different. As seen from Figs. 8 and 9 now there are stable equilibria in the high $P_{a}$-low $R_{0}$ regime where the bubble is both stable towards shape oscillations and fulfills the energy focusing criterion.

In order to predict which SL regime is realized for given $c_{\infty}$ and $P_{a}$, we also have to take the above shape instabilities into consideration. Therefore, in Fig. 10 we plot an enlargement of Fig. 9 together with the thresholds for the shape instabilities and the energy focusing condition, taken from

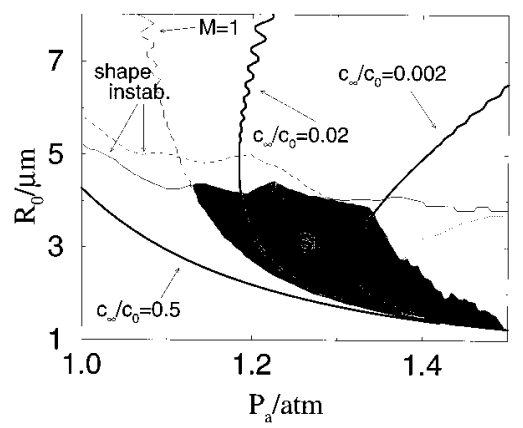

FIG. 10. The figure shows the discussed effects for argon all together: Beyond the $M=1$ curve (long dashed) SL is possible. The bubble grows thanks to rectified diffusion right of the diffusive stability curves (shown for $c_{\infty} / c_{0}=0.5,0.02$, and 0.002 , left to right). At the parametric shape instability (solid) and the shape instability according to criterion (18) (dashed) microbubbles pinch off, but the bubble can survive in the trap for $P_{a} \lesssim 1.4$ atm. SL is possible in the shaded region, stable SL, if in addition the slope of the diffusive equilibrium curve is positive. Here, this occurs for $c_{\infty} / c_{0}=0.002$ for $P_{a} \approx 1.33 \mathrm{~atm}$ and $R_{0} \approx 3 \mu \mathrm{m}$. Right of the dotted line (criterion (21)) the bubble is thrown out of the trap in case of a pinchoff. Consequently, if at all, only stable SL is possible in this high $P_{a}$ regime. 
Fig. 5. In the relevant parameter regime $P_{a}>1.0 \mathrm{~atm}$ the critical $R_{0}^{\mathrm{PI}}$ is about $4-5 \mu \mathrm{m}$. Only for large $P_{a} \gtrsim 1.4 \mathrm{~atm}$ does the Rayleigh-Taylor instability becomes relevant, see below for the consequences. As worked out in Sects. II and III, SL can only occur in the shaded region in Fig. 10. In this region only for very low concentration $c_{\infty} / c_{0} \sim 0.002$ the bubble is diffusively stable and only then can we have diffusively stable SL. For larger concentration we have diffusively unstable SL.

The different regimes of diffusively stable and unstable SL and no SL can easily be read off from Fig. 8. Take fixed $P_{a}=1.1$ : growing bubbles are possible for $c_{\infty} / c_{0}>0.07$, if they are large enough. They finally run into the parametric shape instability at $\sim 5 \mu \mathrm{m}$ and a microbubble will pinch off. If the remaining bubble is still large enough, i.e., above the stability line in Fig. 8, the process will repeat. The allowed size of the bubble after the pinch off is very much restricted; for $c_{\infty} / c_{0}=0.1$ it is only in the $R_{0}$ range between $4.6 \mu \mathrm{m}$ and $5 \mu \mathrm{m}$. If the pinched off microbubble is larger, the remaining bubble will dissolve. No diffusively stable regime exists.

For $P_{a}=1.3$ the situation is quite different. For concentrations in a window $0.003<c_{\infty} / c_{0}<0.005$ bubbles in the $R_{0}$ regime $\sim 2 \mu \mathrm{m}$ up to $5 \mu \mathrm{m}$ will grow and stabilize. As in this $P_{a}$ regime the energy focusing criterion is fulfilled, we have stable SL. Here we are at the very core of Barber's finding ${ }^{5}$ that stable SL is only possible for low $c_{\infty}$ and high $P_{a}$. If the relative concentration is even smaller than 0.003 , bubbles will dissolve and no SL is possible. For larger concentration large enough bubbles $\gtrsim 1.8 \mu \mathrm{m}$ (almost independent of $c_{\infty}$ ) will grow up to $\sim 5 \mu \mathrm{m}$ where microbubbles pinch off. In contrast to smaller $P_{a} \sim 1.1$ atm, the remaining bubble is very likely to end up in the (now much larger) growing $R_{0}$ regime. Pinched off microbubbles with $R_{0}<1.8 \mu \mathrm{m}$ will dissolve.

The same domains no SL, unstable SL, and stable SL can be identified from Fig. 9 or Fig. 10; also these plots make it very evident that high $P_{a} / P_{0}$ and low $c_{\infty} / c_{0}$ are necessary to obtain stable SL.

The total phase diagram in the $c_{\infty}-P_{a}$ phase space, our main result, was already presented in Fig. 1 . The notation in that diagram is as follows: If we denote a regime with stable SL or unstable SL we mean that there are bubbles of certain radius which are diffusively stable or growing, respectively; other, smaller bubbles dissolve. For $P_{a} \lesssim 1.17$ the $c_{\infty} / c_{0}$ window of stable SL shrinks to zero. For very low $c_{\infty}$ the no SL regime (i.e., no bubble regime) is very extended. If one now slightly increases $c_{\infty}$, one immediately enters a regime where the energy focusing condition is fulfilled. This may be the prime reason why it is so much easier to find SL, diffusively stable or not, for low concentration.

The experimental observations of Fig. 4 in Ref. 5 are in agreement with our analysis. For that figure $P_{a}$ is in the range of $1.3 \mathrm{~atm}$. Then we have unstable SL for large concentrations $c_{\infty} / c_{0}=26 \%$ and $6.6 \%$ and stable SL for low concentration $c_{\infty} / c_{0}=0.4 \%$.

What happens for very large forcing amplitudes $P_{a}$ ? From Fig. 10 we see that the SL regime (shaded) intersects with the regime where bubbles cannot survive the microbubble pinchoff (right of the dotted line (21) in Fig. 10) because of the violent short time scale Rayleigh-Taylor instability. Consequently, in this high $P_{a}$ regime only stable SL should be possible, a prediction which is worthwhile being tested experimentally. Extremely low argon concentrations $c_{\infty} / c_{0} \lesssim 0.0006$ are necessary to guarantee diffusively stable equilibria in this regime. According to the approximate curve (21) of the onset of the RT instability in Fig. 10, this regime starts at about $P_{a} \approx 1.4 \mathrm{~atm}$, but as pointed out in Section II D, this number should not be taken too strictly. For lucidity we did not put in the right borderline of the unstable SL regime in the phase diagram Fig. 1. The upper borderline of the unstable SL regime is discussed in Section IV E.

In our former publication ${ }^{42}$ we have stressed the importance of wiggles such as those in the graphs in Figs. 5-10. As a consequence, for large enough $P_{a}$ and small enough $R_{0}$ one may have stable SL with different, discrete $R_{0}$; i.e., multiple stable equilibria. We will present some results of full numerical simulations on this issue in Section V.

Possibly an experimental hint to multiple stable equilibria has been found by Crum and Cordry. ${ }^{51}$ After registering SL light from a bubble for a few seconds, they distorted the bubble and observed the SL intensity to jump from one constant value to another (smaller) one. They suggested that the discrete light intensities corresponded to discrete diffusively stable radii.

For the dynamics chosen here the multiple stable equilibria are just parametrically unstable as they lie beyond $R_{0} \approx 5 \mu \mathrm{m}$. This should not be taken too strictly, as a slight change of the model may allow for observable multiple stable equilibria. However, we see that the wiggles are not necessary for stabilization. An increase of $\langle p\rangle_{t, 4}\left(R_{0}\right)$ with $R_{0}$ is sufficient, i.e., $\beta>0$. If there are wiggles, we in addition have discretization of the equilibria.

\section{Scaling laws}

The average slope and the wiggles in Fig. 7 can be understood in detail as shown in Ref. 47. Here we only quote scaling laws for the running average of $\langle p\rangle_{t, 4}\left(R_{0}\right)$ which smooths out any wiggles,

$$
\overline{\langle p\rangle}_{t, 4}=\frac{1}{2 \Delta R_{0}} \int_{R_{0}-\Delta R_{0}}^{R_{0}+\Delta R_{0}}\langle p\rangle_{t, 4}\left(R_{0}+x\right) d x
$$

with $\Delta R_{0} \geqslant 0.5 \mu \mathrm{m}$. For very small $R_{0}$ the surface tension term dominates in the RP Eq. (2) and $\langle p\rangle_{t, 4} \propto R_{0}^{-1}$. For slightly larger $R_{0}<R_{0}^{\text {crit }}$ we have $\langle p\rangle_{t, 4} \propto R_{0}^{-3 / 2}$. For large $R_{0}>R_{0}^{\text {crit }}$ (where wiggles occur and the running average becomes necessary) the first and the second term on the rhs of (2) balance and

$$
\overline{\langle p\rangle}_{t, 4} \propto R_{0}^{6 / 5},
$$

i.e., the average slope $\beta$ is positive and equilibria are stable. The critical ambient radius $R_{0}^{\text {crit }}$ beyond which wiggles occur and stability is achieved scales like ${ }^{47}$

$$
R_{0}^{\text {crit }} \propto \frac{1}{P_{a}-P_{0}},
$$


the corresponding average pressure like

$$
{\overline{\left\langle p^{\text {crit }}\right.}}_{t, 4} \propto\left(\frac{1}{P_{a}-P_{0}}\right)^{9 / 2} \text {. }
$$

The scaling law (39) directly reflects the average slope $R_{0}$ $\propto c_{\infty}^{5 / 6}$ in Fig. 8. Thus for large enough $R_{0}$ there is always diffusive stabilization, but only for low $c_{\infty}$ and high $P_{a}$ this will be in a parametrically stable domain where the energy focusing condition is fulfilled.

A detailed discussion of these types of scaling laws will be presented elsewhere. ${ }^{47}$

\section{E. Diffusively unstable SL bubbles}

In the previous subsections we saw that our theoretical stability diagram is in agreement with experiment. What about the growth rates in the unstable SL regime where the bubble grows by rectified diffusion and finally hits the parametric instability line at about $R_{0}=5 \mu \mathrm{m}$ ? Because of the growth (i) the relative phase of light emission $\phi\left(R_{0}\right)$ will slightly change, (ii) the light intensity will increase as more and more gas is in the bubble, and (iii) the maximal radius will increase. When the shape instability line is hit, a microbubble pinches off, giving the bubble a recoil. As this repeats again and again on the diffusive time scale of $\sim 1 \mathrm{~s}$, the bubble seems to "dance."

Within the adiabatic approximation we now calculate $\phi\left(R_{0}(\bar{t})\right)=\phi(\bar{t})$. According to the energy focusing mechanisms discussed in Section III light can be emitted if the (inward) bubble wall velocity becomes supersonic, $M=-\dot{R} / c_{\text {gas }} \gtrsim 1$. We define the time when $M=1$ holds as $t_{s}$. The relative shift of this time to the forcing phase defines $\phi_{s}$. As the waves and shock waves in the bubble are very fast, we take the time $t_{s}$ of the detachment of the (shock) wave as the time of the light pulse. The error we make by this approximation is of the order of $\Delta t \sim R_{0} / c_{\mathrm{gas}} \sim 1 \mathrm{~ns}$, as follows from a simple estimate.

Next we calculate $R_{0}(\bar{t})$ from Eq. (35) for discrete adiabatic times $\bar{t}=n T$. Take fixed $R_{0}$ and calculate the time averages $\langle\cdot\rangle_{t, i}$ from the RP equation. The integral

$$
I=\int_{0}^{\infty} \frac{d h^{\prime}}{\left\langle\left(3 h^{\prime}+R^{3}(t)\right)^{4 / 3}\right\rangle_{t, 0}}
$$

can be calculated numerically. Its convergence, however, is slow. In order to speed up the numerical calculation, for calculations over a long period of time we approximate the integral $I$ by $I=a / R_{\max }+(1-a) / R_{0}+\left(3 h_{\max }\right)^{-1 / 3}$. Here, $a \approx 0.9$ is an adjustable parameter, which slightly depends on $R_{0}$ and $P_{a}$, and $h_{\text {max }} \gg R_{\max }^{3}$ must be sufficiently large. The approximation is very well controlled and the results are indistinguishable from the exact result. The growth during the time interval $T$ finally reads

$$
\Delta R_{0}(\bar{t})=\frac{T D c_{0}}{\rho_{0} R_{0}^{2}(\bar{t}) I}\left[\frac{c_{\infty}}{c_{0}}-\frac{\langle p(t)\rangle_{t, 4}(\bar{t})}{P_{0}}\right] .
$$

$\Delta R_{0}$ is added to $R_{0}$ and the procedure is repeated until $R_{0}(\bar{t})$ hits the parametric instability curve. Here from Fig. 5 we took $R_{0}^{\mathrm{PI}}=5 \mu \mathrm{m}$ as a very good approximation. A random fraction of the bubble will pinch off. In Fig. 11 we show the
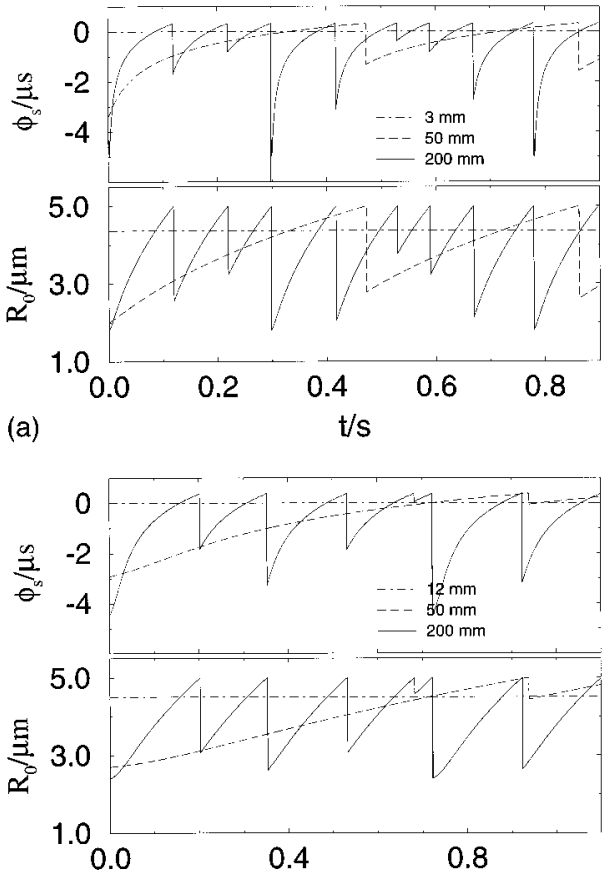

(b)

$\mathrm{t} / \mathrm{s}$

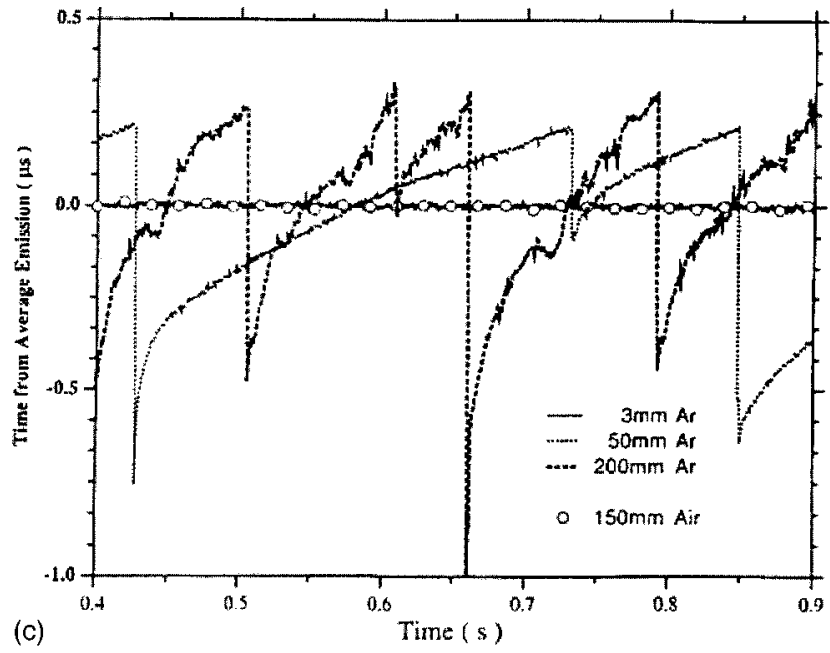

FIG. 11. The phase of the light pulse $\phi_{s}(\bar{t})$ (upper) and the corresponding ambient radius $R_{0}(\bar{t})$ (lower) for (a) $P_{a}=1.3 \mathrm{~atm}$ and for (b) $P_{a}=1.2 \mathrm{~atm}$, for three different gas concentrations $c_{\infty} / c_{0}=0.00395, c_{\infty} / c_{0}=0.0658$, and $c_{\infty} / c_{0}=0.26$, corresponding to a gas pressure of $3 \mathrm{mmHg}, 50 \mathrm{mmHg}$, and $200 \mathrm{mmHg}$, respectively. These values are chosen as in experiment to which these figures compare very well. For $P_{a}=1.2 \mathrm{~atm}$ we had to choose 12 $\mathrm{mmHg}$ as smallest concentration, as for $3 \mathrm{mmHg}$ the bubble would still dissolve. Diffusively stable SL is only seen for the lowest concentration. The strength of the microbubble pinch-off at $R_{0}=5 \mu \mathrm{m}$, i.e., the decrease of the ambient radius, is chosen randomly. (c) Experimental result for the phases of light emission for the same three gas concentrations as in (a). This figure is reproduced from Fig. 4 of Barber et al. (Ref. 5) with kind permission by the authors. It also shows the relative phase of light emission for air bubbles: Stable SL is achieved for much higher concentration $c_{\infty}^{\text {air }} / c_{0}=0.2$, corresponding to $150 \mathrm{mmHg}$. The discrepancy between air and argon can be resolved by also considering chemical instabilities (Refs. 25 and 26).

ambient radius $R_{0}(\bar{t})$ and the relative phase of the light pulse $\phi_{s}(\bar{t})$ for three different relative gas concentrations $c_{\infty} / c_{0}$.

Figure 11 should be compared to the corresponding experimental Fig. 4 of Ref. 5. Unfortunately, for that figure the precise forcing pressure amplitude $P_{a}$ and the ambient 
TABLE I. Growth rates for the phase of light emission $\phi_{s}$ for two forcing pressure amplitudes $P_{a}$ and two concentrations $c_{\infty}$ near the pinch off of the microbubble in comparison with the experimental data (Ref. 5) for which the forcing is not exactly known. Stronger forcing and larger argon concentration enhance the growth. We find order of magnitude agreement. We also give the growth rates for the radii which are not experimentally available.

\begin{tabular}{ccccc}
\hline \hline & $c_{\infty}$ & $P_{a}=1.2 \mathrm{~atm}$ & $P_{a}=1.3 \mathrm{~atm}$ & Experiment $^{5}$ \\
\hline$\phi_{s}$ & $50 \mathrm{mmHg}$ & $1.7 \mu \mathrm{s} / \mathrm{s}$ & $2.7 \mu \mathrm{s} / \mathrm{s}$ & $0.5 \mu \mathrm{s} / \mathrm{s}$ \\
& $200 \mathrm{mmHg}$ & $9 \mu \mathrm{s} / \mathrm{s}$ & $11 \mu \mathrm{s} / \mathrm{s}$ & $5 \mu \mathrm{s} / \mathrm{s}$ \\
$R_{0}$ & $50 \mathrm{mmHg}$ & $2.4 \mu \mathrm{m} / \mathrm{s}$ & $4.7 \mu \mathrm{m} / \mathrm{s}$ & $\cdots$ \\
& $200 \mathrm{mmHg}$ & $11 \mu \mathrm{m} / \mathrm{s}$ & $18 \mu \mathrm{m} / \mathrm{s}$ & $\cdots$ \\
\hline \hline
\end{tabular}

bubble size are not known. So we try both $P_{a}=1.3 \mathrm{~atm}$ (Fig. 11(a)) and $P_{a}=1.2$ atm (Fig. 11(b)). For $P_{a}=1.3$ atm we have very good agreement with experiment: stable SL for 3 $\mathrm{mmHg}$, growth for $50 \mathrm{mmHg}$ and for $200 \mathrm{mmHg}$. For $P_{a}=1.2$ atm a bubble in a fluid with $3 \mathrm{mmHg}$ argon concentration would dissolve according to our approximation, see Fig. 1, so we choose $12 \mathrm{mmHg}$ as smallest concentration and find stable SL.

Let us compare the growth rates with the experimental values. As in experiment, for larger gas concentration the growth rates of $R_{0}$ and $\phi_{s}$ strongly increase. Quantitative estimates from Fig. 11 for the growth rates of the phases for both forcing pressures and both concentrations are compared to the experimental ones in Table I. They are slightly larger from what is found in experiment, but agree in order of magnitude. Rather than focusing on an exact quantitative agreement here, the important point is that for lower concentration $c_{\infty} / c_{0}=0.0165$ (corresponding to $3 \mathrm{mmHg}$ ) the phase of light emission is stable due to the diffusively stable ambient radius (for $P_{a}=1.3 \mathrm{~atm}$ ). In this example, the bubble is locked at $R_{0}=4.31 \mu \mathrm{m}$, and, theoretically, the phase of the light pulse is stable for ever. A practical restriction to this stability is that the cell is not gas tight and additional gas will dissolve, leading to an increase of $c_{\infty}$ which finally leads to bubble destabilization.

What is the physical consequence of the large bubble growth rates obtained for large argon concentration $c_{\infty} / c_{0}$ (Table I)? The shape instability line will be hit more frequently per unit time and the bubble's dancing frequency will thus become larger, as more microbubble pinch-offs and resulting bubble recoils will take place per unit time. As noted above, with a certain probability per pinch-off the pinched off microbubble(s) are too large so that the remaining bubble dissolves. Thus with increased pinch off frequency this probability per time increases. We speculate that this mechanism sets the upper threshold of the unstable SL regime (towards a no SL regime) in the phase diagram Fig. 1. Table I teaches us that the growth rates increase drastically with $c_{\infty} / c_{0}$, so the probability of having a long living bubble for high gas concentration becomes very low. Indeed, Gompf $^{52}$ reported that the larger the concentration is for fixed $P_{a}$, the faster the unstable SL bubble dies. If $c_{\infty}$ is big enough, it will thus be very unlikely for the bubble to survive an appreciable time. From experiment ${ }^{5}$ we know that the upper concentration threshold of unstable SL is beyond $c_{\infty} / c_{0}=0.26$.
This hypothesis also explains why the water in the SL container "ages,",52 in case the container is not gas tight. By "aging" it is meant that stable SL and finally also unstable SL becomes impossible with "old" water. The reason is that external air diffuses into the water, dissolves, and $c_{\infty} / c_{0}$ increases. Consequently, the originally stable bubble is pushed into the unstable regime and starts to "dance," shedding off microbubbles. The dancing frequency becomes higher and higher and finally the bubble dissolves after a too large pinch off. Bubbles may be reseeded, but will also die very soon.

What we do not understand in the unstable regime is the dependence of the light intensity on the gas concentration as e.g., measured in Fig. 2 of Ref. 10. We speculate that it depends on the ambient size of the bubble which is supported by Fig. 6 of Ref. 7. In that figure Löfstedt et al. show that the maximal radius and the SL intensity are correlated.

Finally we address the question whether the growth of $\phi_{s}$ has to be monotonous. In fact, it does not. For larger $R_{0}$ ( $\approx 6 \mu \mathrm{m}$, where the bubble is already parametrically unstable) the growth rate of $\phi_{s}$ is wiggly. Similar oscillations show up in the maximal radius $R_{\text {max }}(t)=\max \left\{R\left(t^{\prime}\right) \mid t \leqslant t^{\prime} \leqslant t+T\right\}$, as e.g., seen in Fig. 4 of Barber et al. ${ }^{3}$ This wiggly structure as a function of time is a direct consequence of the wiggly structure of $\phi_{s}$ and $R_{\max }$ as a function of ambient radius $R_{0}$ which is due to a resonance phenomenon in the RP equation. ${ }^{47}$ The growing $R_{0}$ probes the wiggles in $\phi_{s}\left(R_{0}\right)$ and $R_{\max }\left(R_{0}\right)$.

In experiment no or hardly any oscillatory structure in $\phi_{s}$ is seen. 5 Consequently, the ambient radius $R_{0}$ does not seem to be in the wiggly regime. Thus multiple stable diffusive equilibria may only be important in the shape unstable regime in the $R_{0}-P_{a}$ parameter space. Indeed, the wiggly structure in $R_{\max }\left(R_{0}(\bar{t})\right)^{3}$ is only revealed when boosting the bubble in the unstable SL regime where it becomes shape unstable after a few ms and bursts (cf. Fig. 4 of Ref. 3).

\section{COMPARISON OF THE ADIABATIC APPROXIMATION TO THE FULL NUMERICAL SOLUTION}

\section{A. Concentration profiles}

To compare our results within the adiabatic approximation with the exact solution, we must numerically solve the PDE (26) with the boundary conditions given above. We sketch our numerical method in Appendix A. Here we report on results. We do not consider shape instabilities in this section.

In Fig. 12 we show concentration profiles of the gas outside the bubble during expansion and collapse. We take an argon bubble driven at 1.15 atm close to diffusive equilibrium. The corresponding dynamics of the bubble radius $R(t)$ and the ambient radius $R_{0}(t)$ have already been shown in Figs. 2(b) and 2(c), respectively. Near the bubble radius minimum the concentration gradient at the bubble wall is negative and the bubble ejects gas which accumulates near the bubble wall as the diffusive time scale is slow compared to the bubble motion. When the bubble is reexpanding, it pushes away the accumulated gas together with the fluid. During the expansion phase the concentration $c(R(t), t)$ at 

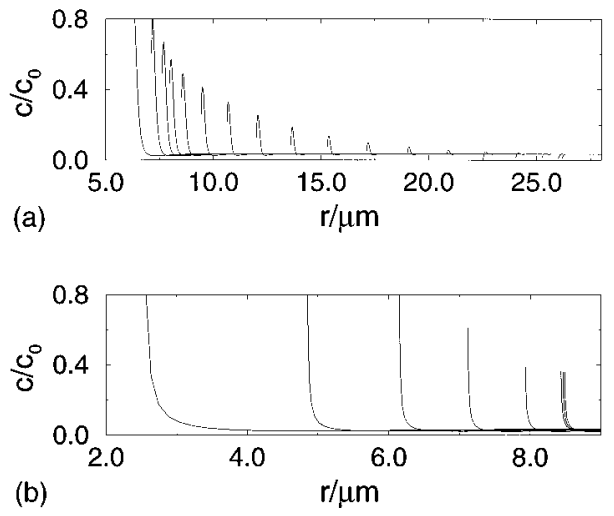

FIG. 12. Gas concentration profiles outside the bubble for expanding (a) and collapsing (b) bubble radius, respectively. The bubble is near diffusive equilibrium, driving pressure is $P_{a}=1.15 \mathrm{~atm}$. Profiles are shown at intervals of $0.75 \mu \mathrm{s}$ (a) and $10 \mathrm{~ns}(\mathrm{~b})$.

the bubble wall decreases due to Henry's law (27). At some point the gradient becomes positive and the mass content of the bubble grows. The wall of gas outside the bubble is thus (i) pushed away from the bubble, (ii) deaccumulates (towards smaller $r$ ) because of bubble growth, and (iii) shrinks because of diffusion (towards larger $r$ ).

Apart from the diffusive processes in the fluid these profiles only mirror the time and space dependence of $c_{\text {osc }}(r, t)$. The width of the boundary layer in which $c_{\text {osc }}$ is a dominant feature of the concentration profile can be readily estimated as

$$
\delta_{D}=\sqrt{\frac{D}{\omega}}
$$

which is the characteristic length for diffusive processes on a time scale $\sim T$.

\section{B. Comparison on diffusive equilibria}

First, let us focus on the equilibrium radii for diffusively stable bubbles. Can we find the multiple equilibria predicted in Section IV in the full numerical simulation, i.e., different stable situations for the same physical parameters? Figure 13 shows $R_{0}(t)$ for several different initial ambient radii. The diffusion constant has the physical value $D=2 \times 10^{-9} \mathrm{~m}^{2} / \mathrm{s}$, corresponding to a Schmidt number of $\mathrm{Sc} \approx 3000$ in the regime of interest $\left(\omega \approx 2 \pi 26.5 \mathrm{kHz}, R_{0} \approx 6 \mu \mathrm{m}\right)$. We indeed observe several stable and unstable equilibria. However, they deviate slightly from those calculated in the adiabatic approximation.

The deviation, however, is tiny, less than $0.06 \mu \mathrm{m}$ as seen from Fig. 14 and clearly not detectable with today's experimental possibilities. Thus for all practical reasons we can consider the adiabatic approximation of the equilibrium radii as exact.

Nevertheless, let us wonder what the origin of the deviation is. It can be explained by considering higher order corrections to the adiabatic theory: ${ }^{53}$ the equilibrium condition (36) is modified, lowering the required mean pressure at the bubble surface for stability to

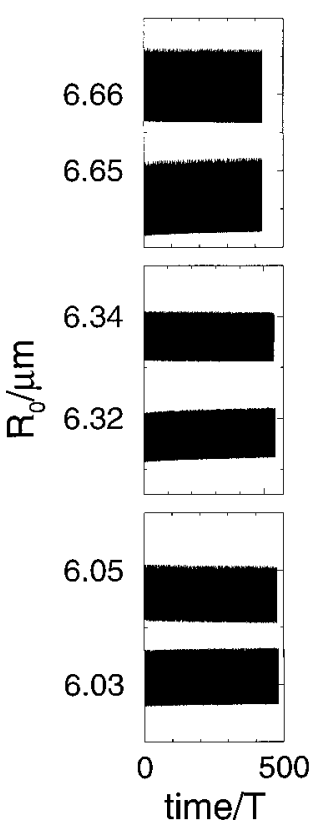

FIG. 13. Three different stable equilibria are approached both from above and from below. Shrinking or growth are hardly noticable. The six initial radii are $6.035 \mu \mathrm{m}, 6.05 \mu \mathrm{m} ; 6.32 \mu \mathrm{m}, 6.34 \mu \mathrm{m} ; 6.65 \mu \mathrm{m}$ and $6.665 \mu \mathrm{m}$, respectively. Otherwise, all conditions are the same. Again, we choose $P_{a}=1.15$ atm. In Fig. 2(c) the same $R_{0}(t)$ dynamics has already been shown for the much shorter time $2 T$. The detailed growth and shrinking processes resolved in that figure cannot be seen here any more as the time scale of $500 T$ chosen here focuses on long time behavior. Therefore, the curves $R_{0}(t)$ seem to "shade" the $R_{0}$ regime between their minimal and their maximal values.

$$
\frac{\langle p(t)\rangle_{t, 4}}{P_{0}}=\frac{c_{\infty}}{c_{0}}-\frac{1}{\mathrm{Sc}^{1 / 2}} \frac{4}{c_{0}}\left\langle\frac{1}{R^{3}} \int_{0}^{\infty} c_{\mathrm{osc}}^{0}(\tilde{h}, t) d \tilde{h}\right\rangle_{t, 4},
$$

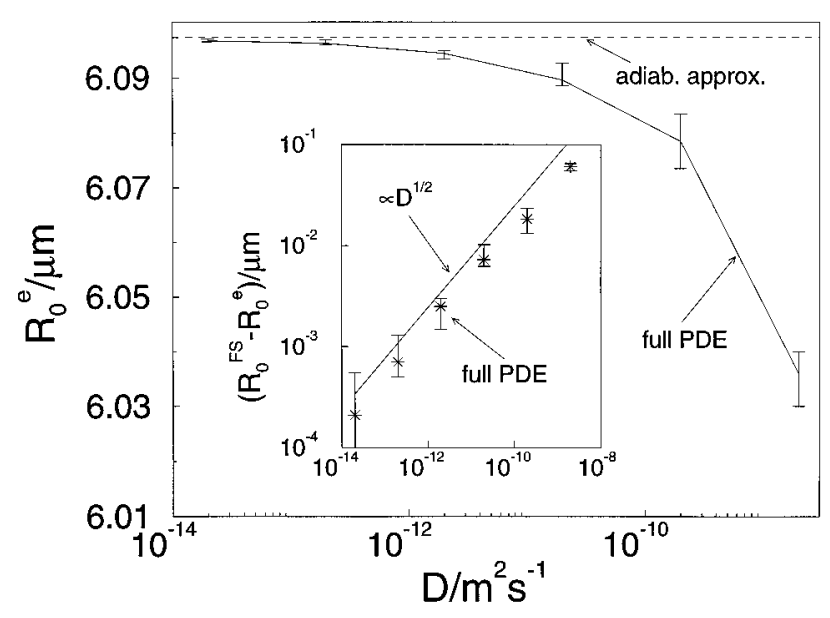

FIG. 14. Location of an equilibrium radius for various $D$ (solid line). At the upper end of the error bar the bubble is shrinking, at the lower end it is growing. The theoretical value from adiabatic approximation is indicated by the dashed line. Note that the tiny difference of $\approx 60 \mathrm{~nm}$ (for the physical diffusion coefficient $D=2 \times 10^{-9} \mathrm{~m}^{2} / \mathrm{s}$ ) is not experimentally detectable at the moment. The bubble is driven at $1.15 \mathrm{~atm}$ with a gas saturation in water of $c_{\infty} / c_{0}=0.035$. The inset shows the deviation $R_{0}^{\text {adiab }}-R_{0}^{e}$ drawn in a loglog diagram, together with our estimate (B1) (solid line). 


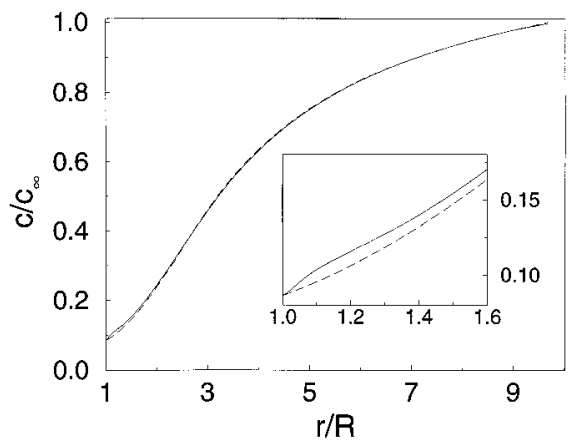

FIG. 15. Comparison of $\langle c(r, t)\rangle_{t, 4}$ (solid line) and the smooth profile $c_{\text {smo }}(r, t)$ from the adiabatic approximation (dashed). The bubble ambient radius shows pronounced growth in this case. The inset shows an enlargement of the small $r$ regime.

$c_{\text {osc }}^{0}$ being the zeroth order (in $\mathrm{Sc}^{-1 / 2}$ ) solution of the oscillatory part of the profile, depending on $\widetilde{h} \equiv \mathrm{Sc}^{1 / 2} h{ }^{53}$ The shift in $\langle p(t)\rangle_{t, 4}$ causes a corresponding shift $R_{0}^{\text {adiab }}-R_{0}^{e}$ in the equilibrium radius $R_{0}^{e}$.

To analyze the deviations from the equilibrium position (36) further, we redid the numerical PDE calculation for smaller (unphysical) $D$. As expected, in the $D \rightarrow 0$ limit the ( $D$ independent) adiabatic fixed point is approached as can be seen from Fig. 14. For that figure the numerical equilibrium radii are "measured" by detecting either "shrinking", or "growth" for slightly different radii. The stable equilibrium is determined by linear interpolation between the growth rates of a growing and a shrinking bubble. In principle, we can achieve arbitrarily small error bars in our numerical results. This is confirmed by the excellent agreement of the $D \rightarrow 0$ limit with the adiabatic approximation.

There is a theoretical possibility that the deviation of the full PDE dynamics from the adiabatic approximation matters, namely when multiple stable equilibria are to be resolved. For completeness we discuss this point in Appendix B.

\section{Comparison on growth rates and profiles}

To detect the location of an equilibrium point along the $R_{0}$ axis, starting with a constant gas concentration profile $c(h)=c_{\infty}$ is obviously a good choice, because $\bar{c}_{\text {smo }}$ will approach that value for all $h$ in equilibrium (apart from higher order corrections). The sign of $\dot{R}_{0}(t)$ will be correct after a small number of oscillation cycles. If one is, however, interested in the actual value of the bubble growth rate, i.e., bubble dynamics far from equilibrium points, choosing $\bar{c}_{\text {smo }}(h)$ as initial concentration profile will avoid transients on diffusive time scales. Indeed,

$$
\langle c(h, t)\rangle_{t, 4} \rightarrow \bar{c}_{\text {smo }}(h)
$$

holds to very good accuracy for such a calculation (see Fig. $15)$. Moreover, the observed growth rate $\dot{R}_{0}(t)$ is in very good agreement with the value calculated from (35), as indicated in Fig. 16.

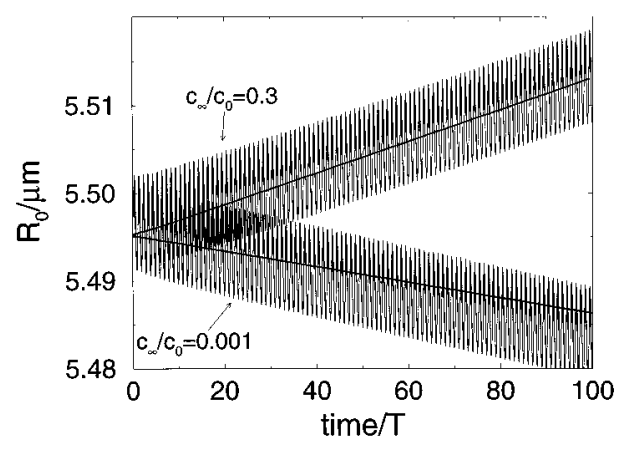

FIG. 16. $R_{0}(t)$ for $c_{\infty} / c_{0}=0.001$ and for $c_{\infty} / c_{0}=0.3$ resulting in shrinking and growth of the bubble, respectively. We chose $P_{a}=1.15 \mathrm{~atm}$ and a bubble with $R_{0}(t=0)=5.5 \mu \mathrm{m}$. The adiabatic approximations are also shown as straight lines. By definition they do not follow the violent mass exchange processes during one cycle of time $T$.

\section{CONCLUSIONS}

This paper works out a hydrodynamic approach towards SL, based on the Rayleigh-Plesset equation. This allows us to explore a considerable part of the phase space and to study long term dynamics. As necessary conditions for SL we demanded shape stability and energy focusing. The adiabatic approximation $^{37,7}$ allows us to study also diffusive stability within the RP approach.

Phase diagrams in the $c_{\infty}-P_{a}, R_{0}-P_{a}$, and $R_{0}-c_{\infty}$ parameter spaces have been presented. Three phases can be identified: stable SL, unstable SL and no SL. Stable SL only occurs in a tiny domain of the parameter space which is in good quantitative agreement with the UCLA SL experiments on argon bubbles. ${ }^{5}$

For molecular gases besides (i) shape instabilities and (ii) diffusive instability also (iii) chemical instabilities have to be considered. Then the present hydrodynamic approach can be extended to gas mixtures such as air as demonstrated in Refs. 25, 26. Again, good agreement with the UCLA experiments ${ }^{4,3,5,7,11}$ is achieved. Our theoretical thresholds of stability may slightly shift if the gas dynamics inside the bubble are more rigorously taken into consideration, e.g., within the acoustic resonator theory ${ }^{23}$ there is an additional pressure caused by the acoustic waves inside the bubble. ${ }^{54}$

We suggest to experimentally map out phase diagrams in the $c_{\infty} / c_{0}$ versus $P_{a} / P_{0}$ parameter space for various gas mixtures. More experimental data on the borderlines between the stable, unstable, and no SL regimes will lead to further refinements and improvements in our understanding of the dynamics of a sonoluminescing bubble. We believe that it is crucial to start a detailed search for discrepancies to the present hydrodynamical (and chemical) theory to judge whether further physical effects which are not considered here play a major role in single bubble sonoluminescence.

The central question which cannot be answered within the present approach is how hot the gas inside the bubble can become. Progress on this point will require more sophisticated understanding of the gas dynamics inside the bubble. Understanding how the gas temperature depends on experimental parameters such as forcing pressure, gas concentration, or liquid temperature will allow for the creation of tem- 
perature controlled environments for chemical reactions within the bubble.

\section{ACKNOWLEDGMENTS}

We thank K. Drese, T. Dupont, W. Eisenmenger, B. Gompf, S. Grossmann, B. Johnston, D. Oxtoby, S. Putterman, and R. Rosales for stimulating discussions and in particular S. Grossmann for continuous encouragement. This work has been supported by the DFG through its SFB 185. M.B. acknowledges a NSF postdoctoral fellowship, and support through the Sloan Research Fund of the School of Science at MIT.

\section{APPENDIX A: NUMERICAL SCHEME FOR THE ADVECTION DIFFUSION EQUATION}

\section{A. Transformation}

To numerically solve Eq. (26) with the boundary conditions (27) and (28) we first transform the independent space variable $r$. Following Ref. 38, we choose

$$
r=F(x, t)=\left((R(t))^{3}-R_{u}^{3} \ln (1-x)\right)^{1 / 3},
$$

where $R_{u}$ is an adjustable length parameter. All lengths are measured as multiples of $1 \mu \mathrm{m}$, all times in units of $T=2 \pi / \omega$, and all pressures in atm. After the transformation $r=F(x, t)$ the advection diffusion equation (26) reads, if written as a local conservation law,

$$
\partial_{t}(f c)+\partial_{x}(g c)-\partial_{x}\left(h \partial_{x} c\right)=0,
$$

where

$$
\begin{aligned}
& f(x, t)=F^{2} \partial_{x} F, \\
& g(x, t)=\dot{R} R^{2}-F^{2} \partial_{t} F, \\
& h(x, t)=D F^{2} / \partial_{x} F .
\end{aligned}
$$

The transformation (A1) is chosen to make $g(x, t)$ identically vanish and thus to obtain a pure diffusion equation. We have

$$
\begin{aligned}
& f(x, t)=R_{u}^{3} /(1-x), \\
& g(x, t)=0, \\
& h(x, t)=D(1-x)(F(x, t))^{4} / R_{u}^{3} .
\end{aligned}
$$

The time dependent range $r \in[R(t), \infty]$ has been mapped to the constant range $x \in[0,1]$. The boundary conditions are Henry's law (27) which now reads $c(x=0, t)=c_{0} p(R, t) / P_{0}$ and $c(x=1, t)=c_{\infty}$. The mass loss of the bubble, expressed in the new independent variable $x$, is

$$
\dot{m}=\left.4 \pi D R_{u}^{-3} R^{4} \partial_{x} c\right|_{x=0} .
$$

\section{B. Discretization}

The interval $x \in[0,1]$ is discretized using a nonequidistant grid. The grid must (i) provide sufficient volume for diffusion of gas outside the bubble and (ii) sufficient resolution near the bubble radius for a correct representation of gas concentration gradients. We satisfy (i) by choosing $R_{u} \approx R_{\max }$, where $R_{\max }$ is the maximum radius of a typical bubble oscillation. This ensures $r^{3}(x \rightarrow 1) \gg R^{3}$ even for relatively low resolution near $x=1$ (cf. Eq. (A1)). From this choice, we deduce a criterion for the grid resolution $\delta x_{R}$ near the bubble surface for a given resolution $\delta r_{R}$ in physical coordinates (e.g., $\delta r_{R}=0.01 \mu \mathrm{m}$ ). Expanding (A1) in $x \ll 1$, we get

$$
\delta x_{R}=\delta r_{R} \frac{R_{\min }^{2}}{R_{\max }^{3}} .
$$

This is a conservative estimate, requiring $\delta r_{R}$ to be resolved even for the minimum bubble radius $R_{\min }$. At times when the bubble radius is larger, the resolution in $r$ will be even better with this $\delta x_{R}$.

Typical bubble dynamics data lead to $\delta x_{R} \approx 5 \times 10^{-6}$. Excellent resolution at the bubble radius is needed for a correct representation of gas concentration gradients. It is, however, unnecessary at greater distance from the bubble surface. Therefore, the grid density is varied according to a power law to yield a fine grid near $x=0$ and a coarse grid near $x=1$.

Overall, we have $N$ grid points $x_{1}=0, x_{2}, \ldots, x_{N-1}$, $x_{N}=1$. The field $c(x, t)$ is defined on the grid, $c_{i}=c\left(x_{i}\right)$, and so are the fields $f, g, h$. We define $d x_{i}=x_{i+1}-x_{i}$, $i=1,2, \ldots, N-1$ and $d x_{a, i}=\left(d x_{i-1}+d x_{i}\right) / 2, i=2, \ldots, N-2$, $d x_{a, 1}=d x_{1} / 2, d x_{a, N}=d x_{N-1} / 2$.

The discretization of Eq. (A2) has to guarantee mass conservation,

$$
\begin{aligned}
0= & c_{1}^{\theta}-p\left(R^{\theta}(t)\right) c_{0} / P_{0}, \\
0= & f_{i} \frac{d c_{i}}{d t}-\frac{1}{d x_{a, i}}\left(\frac{1}{2}\left(h_{i}^{\theta}+h_{i+1}^{\theta}\right) \frac{c_{i+1}^{\theta}-c_{i}^{\theta}}{d x_{i}}\right. \\
& \left.-\frac{1}{2}\left(h_{i-1}^{\theta}+h_{i}^{\theta}\right) \frac{c_{i}^{\theta}-c_{i-1}^{\theta}}{d x_{i-1}}\right), \quad i=2, \ldots, N-2 \\
0= & f_{i} \frac{d c_{i}}{d t}-\frac{1}{d x_{a, i}}\left(-\frac{1}{2}\left(h_{i-1}^{\theta}+h_{i}^{\theta}\right) \frac{c_{i}^{\theta}-c_{i-1}^{\theta}}{d x_{i-1}}\right), \\
& i=N-1, \\
0= & c_{N}^{\theta}-c_{\infty} .
\end{aligned}
$$

Here, $c_{i}^{\theta}$ are the concentrations $c_{i}+\theta d c_{i}$ at time $t+\theta d t$ and $h_{i}^{\theta}=h_{i}\left(R^{\theta}\right)$ where $R^{\theta}$ is the radius at time $t+\theta d t$. Correspondingly, $R_{0}^{\theta}$ is the ambient radius at that time. We choose $\theta=1$, i.e., a fully implicit method. Equation (A6) has to be assisted by the Rayleigh-Plesset Eq. (2) and the proper discretization of (A5) guaranteeing total mass conservation,

$$
0=\rho_{0}\left(R_{0}^{\theta}\right)^{2} \dot{R}_{0}^{\theta}-\frac{h_{1}^{\theta}+h_{2}^{\theta}}{2} \frac{c_{2}^{\theta}-c_{1}^{\theta}}{d x_{1}} .
$$

We solve the $N+2$ equations (A6), (A7), and (2) for the unknowns $d c_{i}, i=1,2, \ldots, N, d R_{0}$, and $d R$ with Newton's method. The Jacobian is calculated analytically. A time step control and adjustment is provided by redoing every time step $d t$ in two steps of width $d t / 2$ each and then comparing the result. For large forcing we need a very low tolerance of $10^{-5}$ per cent to achieve sufficient numerical quality of mass 
conservation. Note that this simulation covers time scales from picoseconds (for good resolution of the bubble dynamics near the collapse) to tens of milliseconds (for observation of diffusive growth or shrinking of the bubble).

\section{APPENDIX B: ADIABATIC APPROXIMATION AND MULTIPLE EQUILIBRIA}

In Section $\mathrm{V}$ we showed that the adiabatic approximation well describes the full dynamics. However, for (theoretical) completeness we would like to caution in this Appendix: For the physical diffusion coefficient $D=2 \times 10^{-9} \mathrm{~m}^{2} / \mathrm{s}$ the simple adiabatic approximation may lead to even qualitatively wrong results, e.g., take a bubble with $R_{0}=6.06 \mu \mathrm{m}$. According to the adiabatic approximation one would expect that it grows towards the equilibrium $R_{0}^{\text {adiab }}=6.097 \mu \mathrm{m}$ (see Fig. 14). However, it shrinks towards the physical equilibrium $R_{0}^{e}=6.041 \mu \mathrm{m}$. At the moment the experimental accuracy does not allow us to distinguish between these two sizes, but it may improve one day. We can understand $R_{0}^{e}<R_{0}^{\text {adiab }}$ because the gas layer around the bubble (Fig. 12) diffusively shrinks too strongly for finite $D$, leading to a larger overall mass loss and thus smaller bubbles. The order of magnitude of the $R_{0}^{e}$ shift can be estimated as the width of the boundary layer:

$$
R_{0}^{\text {adiab }}-R_{0}^{e} \sim \delta_{D}=\sqrt{\frac{D}{\omega}} \propto D^{1 / 2}
$$

For the physical $D=2 \times 10^{-9} \mathrm{~m}^{2} / \mathrm{s}$ we have $R_{0}^{\text {adiab }}-R_{0}^{e} \approx 0.11 \mu \mathrm{m}$ reproducing the numerical result $\approx 0.06 \mu \mathrm{m}$ quite accurately. In the inset of Fig. 14 we plot $\log \left(R_{0}^{\text {adiab }}-R_{0}^{e}\right)$ vs $\log D$ and indeed find good agreement with the scaling law (B1).

Whether the deviations are considered to be serious or not depends on what is supposed to be analyzed. If the focus lies on identifying equilibrium points, the consequences can be quite drastic. Note that for large enough $D$, the value of (45) falls below the minimum values of $\langle p(t)\rangle_{t, 4} / P_{0}$ (cf. Fig. $7)$, thus making an equilibrium solution impossible and leading to dissolution of the bubble.

If the distance between subsequent equilibrium radii $\Delta R_{0}^{\text {adiab }, i} \equiv R_{0}^{\text {adiab }, i}-R_{0}^{\text {adiab, } i-1}$ (where the index numbers label the equilibria in ascending $R_{0}$ order) is supposed to be resolved, i.e., if one wants to find a one to one correspondence between the adiabatic equilibria and the real ones, one has as to impose the following condition on the approximation:

$$
\Delta R_{0}^{\text {adiab }, i} \gg \frac{1}{2}\left(R_{0}^{\text {adiab }, i}-R_{0}^{e}\right) \sim \frac{\delta_{D}}{2} .
$$

Expressed in the Schmidt number Sc it reads

$$
\mathrm{Sc} \gg\left(\frac{2 R_{0}}{\Delta R_{0}^{\text {adiab }, i}}\right)^{2}
$$

rather than simply $\mathrm{Sc} \gg 1$ as one may naively expect. With the correct values in the relevant parameter regime $R_{0} \approx 6 \mu \mathrm{m}, \Delta R_{0}^{\text {adiab, } i} \approx 0.3 \mu \mathrm{m}$ we have $\mathrm{Sc} \gg 1600$, which is only marginally fulfilled by the physical $D$ corresponding to $\mathrm{Sc} \approx 3000$.
${ }^{1}$ D. F. Gaitan, "An experimental investigation of acoustic cavitation in gaseous liquids," Ph.D. thesis, The University of Mississippi, 1990; D. F. Gaitan, L. A. Crum, R. A. Roy, and C. C. Church, "Sonoluminescence and bubble dynamics for a single, stable, cavitation bubble," J. Acoust. Soc. Am. 91, 3166 (1992).

${ }^{2}$ B. P. Barber and S. J. Putterman, "Observation of synchronous picosecond sonoluminescence," Nature (London) 352, 318 (1991); "Light scattering measurements of the repetitive supersonic implosion of a sonoluminescing bubble,"' Phys. Rev. Lett. 69, 3839 (1992).

${ }^{3}$ B. P. Barber, C. C. Wu, R. Löfstedt, P. H. Roberts, and S. J. Putterman, "Sensitivity of sonoluminescence to experimental parameters," Phys. Rev. Lett. 72, 1380 (1994).

${ }^{4}$ R. Hiller, K. Weninger, S. J. Putterman, and B. P. Barber, "Effect of noble gas doping in single-bubble sonoluminescence," Science 266, 248 (1994). ${ }^{5}$ B. P. Barber, K. Weninger, R. Löfstedt, and S. J. Putterman, "'Observation of a new phase of sonoluminescence at low partial pressures,' Phys. Rev. Lett. 74, 5276 (1995).

${ }^{6}$ R. Löfstedt, B. P. Barber, and S. J. Putterman, "Toward a hydrodynamic theory of sonoluminescence," Phys. Fluids A 5, 2911 (1993).

${ }^{7}$ R. Löfstedt, K. Weninger, S. J. Putterman, and B. P. Barber, "Sonoluminescing bubbles and mass diffusion," Phys. Rev. E 51, 4400 (1995).

${ }^{8}$ R. G. Holt, D. F. Gaitan, A. A. Atchley, and J. Holzfuss, "Chaotic sonoluminescence,' Phys. Rev. Lett. 72, 1376 (1994).

${ }^{9}$ M. J. Moran et al., "Direct observations of single sonoluminescence pulses,' Nucl. Instrum. Methods in Phys. Res. B 96, 651 (1995).

${ }^{10}$ K. Weninger, R. Hiller, B. P. Barber, D. Lacoste, and S. J. Putterman, "Sonoluminescence from single bubbles in non-aqueous liquids: new parameter space for sonochemistry," J. Phys. Chem. 99, 14195 (1995).

${ }^{11}$ R. Hiller and S. J. Putterman, "Observation of isotope effects in sonoluminescence," Phys. Rev. Lett. 75, 3549 (1995).

${ }^{12}$ M. S. Plesset, "On the stability of fluid flows with spherical symmetry," J. Appl. Phys. 25, 96 (1954).

${ }^{13}$ G. Birkhoff, "Note on Taylor instability," Q. Appl. Math. 12, 306 (1954).

${ }^{14} \mathrm{~A}$. Eller and L. A. Crum, "Instability of the motion of a pulsating bubble in a sound field," J. Acoust. Soc. Am. 47, 762 (1970).

${ }^{15} \mathrm{H}$. W. Strube, "Numerische Untersuchungen zur Stabilitát nichtsphärisch schwingender Blasen," Acustica 25, 289 (1971).

${ }^{16}$ A. Prosperetti, "Viscous effects on perturbed spherical flows," Q. Appl. Math. 34, 339 (1977).

${ }^{17}$ M. P. Brenner, D. Lohse, and T. F. Dupont, "Bubble shape oscillations and the onset of sonoluminescence," Phys. Rev. Lett. 75, 954 (1995).

${ }^{18}$ J. Schwinger, "Casimir energy for dielectrics spherical geometry," Proc. Natl. Acad. Sci. U.S.A. 89, 11118 (1992).

${ }^{19}$ E. B. Flint and K. S. Suslick, "Sonoluminescence from nonaqueous fluids: emission from small molecules," J. Am. Chem. Soc. 111, 6987 (1989).

${ }^{20}$ P. Jarman, "Sonoluminescence: A discussion,', J. Acoust. Soc. Am. 32, 1459 (1960).

${ }^{21}$ H. P. Greenspan and A. Nadim, "On sonoluminescence of an oscillating gas bubble," Phys. Fluids A 5, 1065 (1993).

${ }^{22} \mathrm{C}$. C. Wu and P. H. Roberts, "Shock-wave propagation in a sonoluminescing gas bubble," Phys. Rev. Lett. 70, 3424 (1993); “A model of sonoluminescence,'” Proc. R. Soc. London, Ser. A 445, 323 (1994).

${ }^{23}$ M. P. Brenner, R. Rosales, S. Hilgenfeldt, and D. Lohse, "Acoustic energy storage in single bubble sonoluminescence," to appear in Phys. Rev. Lett.

${ }^{24}$ L. D. Landau and E. M. Lifshitz, Fluid Mechanics (Pergamon Press, Oxford, 1987).

${ }^{25}$ D. Lohse, M. P. Brenner, T. F. Dupont, S. Hilgenfeldt, and B. Johnston, "Sonoluminescing air bubbles rectify argon," preprint, September 1996.

${ }^{26}$ M. P. Brenner, S. Hilgenfeldt, and D. Lohse, "Why air bubbles in water glow so easily," in Nonlinear Physics of Complex Systems-Current Status and Future Trends, edited by J. Parisi, S. C. Müller, and W. Zimmermann (Springer, Berlin, 1996).

${ }^{27}$ Lord Rayleigh, "'On the pressure developed in a liquid on the collapse of a spherical bubble," Philos. Mag. 34, 94 (1917).

${ }^{28}$ M. S. Plesset, "The dynamics of cavitation bubbles," J. Appl. Mech. 16, 277 (1949).

${ }^{29} \mathrm{G}$. I. Taylor, "The instability of liquid surfaces when accelerated in a direction perpendicular to their planes I," Proc. R. Soc. London, Ser. A 201, 192 (1950).

${ }^{30} \mathrm{~W}$. Lauterborn, "Numerical investigation of nonlinear oscillations of gas bubbles in liquid," J. Acoust. Soc. Am. 59, 283 (1976).

${ }^{31}$ M. S. Plesset and A. Prosperetti, "Bubble dynamics and cavitation", Annu. Rev. Fluid Mech. 9, 145 (1977). 
${ }^{32}$ J. B. Keller and M. J. Miksis, "Bubble oscillations of large amplitude," J. Acoust. Soc. Am. 68, 628 (1980); B. E. Noltingk and E. A. Neppiras, "Cavitation produced by ultrasonics," Proc. Phys. Soc. London B 63, 674 (1950); E. A. Neppiras and B. E. Noltingk, "Cavitation produced by ultrasonics: theoretical conditions for the onset of cavitation," ibid. B 64, 1032 (1951). Further references can be found in Brennen's book (Ref. 33).

${ }^{33}$ C. E. Brennen, Cavitation and Bubble Dynamics (Oxford University Press, Oxford, 1995)

${ }^{34}$ P. S. Epstein and M. S. Plesset, "'On the stability of gas bubbles in liquidgas solutions," J. Chem. Phys. 18, 1505 (1950).

${ }^{35} \mathrm{~A}$. Eller and L. A. Crum, "Instability of the motion of a pulsating bubble in a sound field,' J. Acoust. Soc. Am. 47, 762 (1970).

${ }^{36}$ L. A. Crum, "Sonoluminescence,"' Phys. Today 47, 22 (1994).

${ }^{37}$ M. M. Fyrillas and A. J. Szeri, "'Dissolution or growth of soluble spherical oscillating bubbles," J. Fluid Mech. 277, 381 (1994).

${ }^{38}$ V. Q. Vuong and A. J. Szeri, "Sonoluminescence and diffusive transport,' Phys. Fluids 8, 2354 (1996).

${ }^{39}$ W. C. Moss, D. B. Clarke, J. W. White, and D. A. Young, "Hydrodynamic simulations of bubble collapse and picosecond sonoluminescence,", Phys. Fluids 6, 2979 (1994); L. Kondic, J. I. Gersten, and C. Yuan, "Theoretical studies of sonoluminescence radiation: radiative transfer and parametric dependence,'’ Phys. Rev. E 52, 4976 (1995).

${ }^{40}$ The recent calculations of Vuong and Szeri (Ref. 38) incorporate dissipation mechanisms and do not find shocks.

${ }^{41}$ V. Kamath, A. Prosperetti, and F. N. Egolfopoulos, "A theoretical study of sonoluminescence,"' J. Acoust. Soc. Am. 94, 248 (1993).

${ }^{42}$ M. P. Brenner, D. Lohse, D. Oxtoby, and T. F. Dupont, "Mechanisms for stable single bubble sonoluminescence," Phys. Rev. Lett. 76, 1158 (1996).

${ }^{43}$ A. Eller and H. G. Flynn, "Rectified diffusion during nonlinear pulsations of cavitation bubbles," J. Acoust. Soc. Am. 37, 493 (1964).
${ }^{44}$ G. K. Batchelor, An Introduction to Fluid Dynamics (Cambridge University Press, Cambridge, 1970).

${ }^{45}$ For a passive scalar as e.g., the gas concentration the analogous length scale is $\sqrt{D / \omega}$, where $D$ is the diffusion constant. In Section $\mathrm{V}$ we perform a full numerical simulation for the diffusive problem and indeed see that $\sqrt{D / \omega}$ is the thickness of the boundary layer around the bubble.

${ }^{46} \mathrm{~A}$ very similar reasoning was applied to the (thermal) boundary layer around the probe measuring the temperature in turbulent helium, see $\mathrm{S}$. Grossmann and D. Lohse, "Characteristic scales in Rayleigh-Benard turbulence," Phys. Lett. A 173, 58 (1993).

${ }^{47}$ S. Grossmann, S. Hilgenfeldt, D. Lohse, and M. P. Brenner, "Analysis of the Rayleigh-Plesset bubble dynamics for large forcing pressure," in preparation, September 1996.

${ }^{48}$ F. G. Blake, J. Acoust. Soc. Am. 21, 551 (1949); V. Bjerknes, Die Kraftfelder (Friedrich Vieweg, Braunschweig, 1909).

${ }^{49}$ G. Guderley, "Starke kugelige und zylindrische Verdichtungsstösse in der Nähe des Kugelmittelpunktes bzw. der Zylinderachse," Luftfahrtforsch. 19, 302 (1942).

${ }^{50}$ E. J. Hinch, Perturbation Methods (Cambridge University Press, Cambridge, 1991).

${ }^{51}$ L. A. Crum and S. Cordry, "Single bubble sonoluminescence," in Bubble Dynamics and Interface Phenomena, edited by J. Blake et al. (Kluwer Academic, Dordrecht, 1994), p. 287.

${ }^{52}$ B. Gompf (private communication, 1996).

${ }^{53}$ M. M. Fyrillas and A. J. Szeri, "Dissolution or growth of soluble spherical oscillating bubbles: The effect of surfactants,' J. Fluid Mech. 289, 295 (1995).

${ }^{54}$ M. P. Brenner, S. Hilgenfeldt, and D. Lohse, "Phase locking in single bubble sonoluminescence,', preprint, September 1996. 